\title{
Combining a distributed flow manifold and 3D woven metallic lattices to enhance fluidic and thermal properties for heat transfer applications
}

Longyu Zhao ${ }^{a,}$, Stephen M. Ryan ${ }^{a}$, Sen Lin ${ }^{b}$, Ju Xue ${ }^{a}$, Seunghyun Ha ${ }^{\text {b, c }}$, Takeru Igusa ${ }^{b}$,

Keith W. Sharp ${ }^{\text {d, James K. Guest }}{ }^{\text {b, a }}$, Kevin J. Hemker ${ }^{\text {e, a }}$, Timothy P. Weihs ${ }^{\text {a, e }}$

${ }^{a}$ Department of Materials Science and Engineering, Johns Hopkins University, 3400 N. Charles Street, Baltimore, MD 21218, USA

${ }^{\mathrm{b}}$ Department of Civil Engineering, Johns Hopkins University, 3400 N. Charles Street, Baltimore, MD 21218, USA

${ }^{c}$ Department of Ocean Engineering, Korea Maritime and Ocean University, 727 Taejong-ro, Yeongdo-Gu, Busan 606-791, Republic of Korea

${ }^{\mathrm{d}}$ SAERTEX USA, LLC., Huntersville, NC 28078, USA

${ }^{\mathrm{e}}$ Department of Mechanical Engineering, Johns Hopkins University, 3400 N. Charles Street, Baltimore, MD 21218, USA

\section{Corresponding Author:}

Longyu Zhao

Johns Hopkins University, Department of Materials Science and Engineering

3400 N. Charles Street, Maryland Hall 205, Baltimore, MD 21218, USA

E-mail: longyu@jhu.edu

Tel: +1 (410)516-4274

Fax: $+1(410) 516-5293$ 


\section{Abstract}

The fluidic and heat transfer capabilities of 3D woven lattice materials were reported recently under axial and bifurcated flow patterns, but three critical performance indices - pressure drop, average surface temperature and temperature uniformity - could not be optimized simultaneously using these flow patterns. Here we combine the 3D weaves with manifolds to create a novel 3D flow pattern that enhances temperature uniformity, while also maintaining low pressure drops and surface temperatures. These three properties were characterized at room temperature for a range of flow rates using water as the working fluid. Three different weaves thicknesses were investigated: $12.7 \mathrm{~mm}, 6.4 \mathrm{~mm}$, and $3.2 \mathrm{~mm}$, with manifold thicknesses of 12.7 $\mathrm{mm}, 19.0 \mathrm{~mm}$, and $22.2 \mathrm{~mm}$, respectively, to provide a constant, combined weave-manifold thickness of $25.4 \mathrm{~mm}$. The properties of this new weave/manifold system are compared to those obtained using just the manifold (with no weave) and just the weave (with no manifold). Comparisons show that the addition of the weave lowers the average substrate temperature and temperature variations significantly, although pressure drop is increased. They also show that the addition of the manifold improves temperature uniformity significantly, and also lowers the average substrate temperature and the pressure drop. No specific ratio of weave to manifold thickness was found to be superior in all of the performance indices. The thermal performances are then evaluated at different pumping powers: the weave/manifold system and its distributed array flow pattern prevail. Finite element simulations were performed on a reduced and simplified model to explain the observed experimental trends, and manifold opening patterns were manipulated to demonstrate further potential property enhancements. The multiple benefits of this manifold system can be extended to common heat exchanger media beyond weaves. 


\section{Keywords}

3D woven lattice materials, flow manifold, distributed array, topology optimization, heat exchanger

\section{Nomenclature}

$\triangle P \quad$ pressure drop $[\mathrm{Pa}]$

$T_{S} \quad$ average surface temperature $[\mathrm{K}]$
$\Delta T$ temperature variation across the surface $[\mathrm{K}]$

$X, Y, Z \quad$ Cartesian coordinates $[\mathrm{m}]$

\section{Introduction}

Recently we reported excellent fluidic and thermal properties for multifunctional 3D woven lattice materials. Due to the pore structure of these lattices, different global fluid flow patterns could be utilized to tailor various response properties, including total heat transfer, substrate temperature uniformity, and pressure drop. Specifically, we considered three flow patterns as shown in Fig. 1: axial, where fluid flows parallel to the substrate; full bifurcated, where the inlet flow is perpendicular to the substrate and turns 90 degrees to the outlet ports; and focused bifurcated, which is similar to the full bifurcated case but the inlet is concentrated over a small region [1]. Among the three applied flow patterns, we found that under the same power inputs $\left(10.3,20.7\right.$, and $\left.31.0 \mathrm{~W} / \mathrm{cm}^{2}\right)$ and volumetric flow rates $\left(1-17 \times 10^{-5} \mathrm{~m}^{3} / \mathrm{s}\right)$, the axial flow transfers heat more effectively than the other two, while the bifurcated flow patterns provided better temperature uniformity and lower pressure drops. Thus, neither flow pattern is ideal in 
terms of these three properties. However, the bifurcated flow patterns have two advantages: increasing the flow rate lowers the average surface temperatures without generating the large temperature variations attendant with flow rate increases in the axial pattern, and the pressure drops in the bifurcated cases are smaller than in the axial cases.

(a)

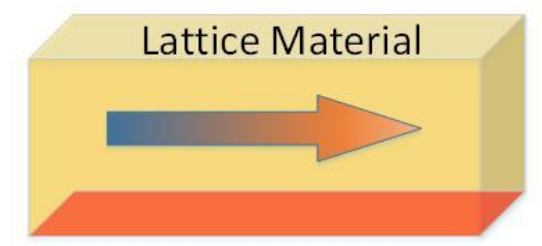

Heated Substrate (b)

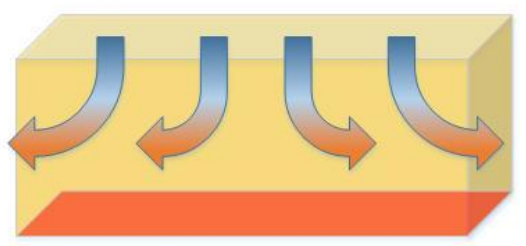

(c)

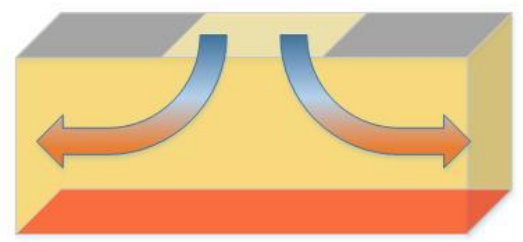

Fig. 1. Schematic of three flow patterns, (a) axial, (b) full bifurcated and (c) focused bifurcated, through the 3D woven lattice material in a previous study [1].

To improve properties beyond those reported for these ordinary flow patterns, we focus here on modifying the bifurcated flow pattern with a manifold to overcome its two main disadvantages. One is that a large portion of the fluid enters and exits the $3 \mathrm{D}$ weave near its edges in bifurcated flow and does not interact sufficiently with the weave to facilitate forced convection between the heated solid and the cooling fluid. A manifold combined with a thinner weave forces more of the fluid flow towards the heated substrate, thereby enhancing heat transfer. Another is that the maximum and minimum temperatures of the heated substrate occur at the center and the edges of the bifurcated flow pattern, respectively, meaning that temperature variations scale with the lateral dimensions of the flow pattern. The use of periodic manifolds can reduce the scale of the bifurcated flow and thereby minimize temperature variations, while 
also accommodating large substrates. Thus, we argue that the combination of a thin metallic weave and a manifold provides the ability to tune local temperature variations for any size substrate while also providing superior fluidic and thermal properties.

Earlier studies have shown that continuous jet impingement of a liquid or gas onto a surface is more effective at removing heat from that surface than forced convection [2-4]. Highspeed jet impingement on a component surface creates a thin boundary layer and enables superior heat transfer. Macro and micro-scale jets [2] have been studied with multiple fluids and flow schemes and they have been used to quench metals and to cool turbine blades. Many practical applications employ an array of jets that typically maintains a more consistent surface temperature and can cool very large areas compared to a single jet. Recently, Motakef et al. described the achievement of heat transfer coefficients of 500,000 and $20,000 \mathrm{~W} / \mathrm{m}^{2} \mathrm{~K}$ with submillimeter, microjet arrays using water and air, respectively [3].

While jet impingement devices have shown tremendous success [4], most of these studies have been performed with the jets spraying fluid onto a substrate through an empty spacing so as to quantify parameters such as heat transfer coefficients or Nusselt numbers while changing variables such as Reynolds number, jet diameter, radial position, wall-to-nozzle spacing, etc. $[5,6]$. By inserting a heat exchange medium, such as a metallic weave, between the jets and the heated substrate, heat transfer can be enhanced further. The solid ligaments of the weave provide high heat conduction and more surface area for improved heat convection, and the local, regular pores enhances fluid mixing. Here we present fluidic and thermal studies of a combined heat exchange system that includes a plastic flow manifold and a 3D $\mathrm{Cu}$ weave. The manifold is designed so that the return or exhaust fluid flow does not interfere with the impinging jets, and its thickness is increased from $12.7 \mathrm{~mm}$ to $19.0 \mathrm{~mm}$ and then to $22.2 \mathrm{~mm}$ while the thickness of 
the weaves is decreased from $12.7 \mathrm{~mm}$ to $6.4 \mathrm{~mm}$ and then to $3.2 \mathrm{~mm}$ to maintain a constant total system thickness of $25.4 \mathrm{~mm}$. We then perform finite element simulations on a reduced and simplified model to study the trends observed in the experiments. For comparison, we also report on the use of only the flow manifold for cooling. Pressure drops, average surface temperatures, and temperature variations are reported and are compared to earlier data for axial and full bifurcated flow patterns within the same $\mathrm{Cu}$ weaves. Finally, methods for optimization of the manifold are suggested to improve the system's performance and one example of adjusting the manifold opening pattern is reviewed.

\section{Experimental procedures and simulation setup}

A schematic of the fluid flow patterns and photographs of the 3D printed plastic flow manifolds are shown in Fig. 2. The coolant first flows through an array of inlet channels that are shown in Fig. 2c and span the manifold's full thickness. Then the coolant flows through the weave and impinges on the heated substrate as an array of high velocity jets (blue arrows in Fig. 2a). Next the coolant returns through the weave and enters a second set of channels shown in Fig. 2 d. These exhaust channels are located between the inlet channels on the bottom surface of the manifold (Fig. 2d) and span only its bottom lip. Thus, the heated, exhaust coolant flows within the hollow structure towards the periphery of the flow manifold where it is collected and eventually leaves via the smaller faces on the right and left of the manifold (orange arrows in Fig. 2a). Alternatively, one could adjust the outlet piping allowing the heated coolant to exit the manifold through the larger faces on the front and back, or through both sets of faces. The

localized exhausting of each jet to the center of the manifold minimizes cross-flow between 
neighboring jets within the weave and near the heated surface. This increases cooling efficiency and also allows the performance of a single jet to be replicated over a large area.

The two different architectures of the 3D woven lattice materials were studied, and both have proven effective in dissipating heat [1]. Details of the lattices, which were composed of 202 micron diameter copper $(\mathrm{Cu})$ wires, and details of the 3D weave manufacturing process can also be found in [1]. One woven architecture is called "standard" and has a dense pattern in which every possible wire position is occupied. The other architecture is called "optimized" and is a more porous pattern in which some wires were selectively eliminated in two Cartesian directions to enhance fluid permeability with minimal reductions in mechanical stiffness $[7,8]$. The selection of wires for removal was guided by topology optimization. Schematics and images of the manufactured woven blocks are shown in Fig. 3a and 3b, for both architectures. To accommodate the testing fixture, the mating areas of the manifold and weave both measure 76.2 $\mathrm{x} 25.4 \mathrm{~mm}$ in the $\mathrm{X}$ and $\mathrm{Z}$ directions and the combined thickness of the manifold and weave is $25.4 \mathrm{~mm}$ in the $\mathrm{Y}$ direction. To study the impact of the ratio of thicknesses for the weave and the manifold, both the standard and optimized weaves were sliced using electrical discharge machining (EDM) to three thicknesses: $3.2 \mathrm{~mm}, 6.4 \mathrm{~mm}$, and $12.7 \mathrm{~mm}$, and they were independently brazed to a $1 \mathrm{~mm}$ face sheet. Three manifolds were then 3D printed in ABS plastic with thicknesses designed to reach a total thickness of $25.4 \mathrm{~mm}$ when combined with one of the three weaves. We note $3 \mathrm{D}$ printed $\mathrm{ABS}$ was chosen due to its low cost and low thermal conduction, ensuring heat transfer to be focused in the woven material. To seal any gaps between the manifold and weave, due to potential uneven contact surfaces, a $1.5 \mathrm{~mm}$ rubber gasket was embedded between them with holes cut to match the hole pattern found on the manifold's bottom surface. The thickness of the rubber gasket is part of the total $25.4 \mathrm{~mm}$ 
thickness. Fig. 3c shows an example of the $6.4 \mathrm{~mm}$ thick weave, the gasket and the manifold with corresponding thicknesses. All components were then stacked and placed into the testing chamber (Fig. 3d), which was described in a previous study [1]. Finally, to measure surface temperatures, the weaves with $\mathrm{Cu}$ face sheets were soldered to the $\mathrm{Cu}$ heat source and seven thermocouples were placed evenly spaced along the length of the heater less than $1 \mathrm{~mm}$ from the bonding surface through thin, blind holes, as shown in Fig. 3e.
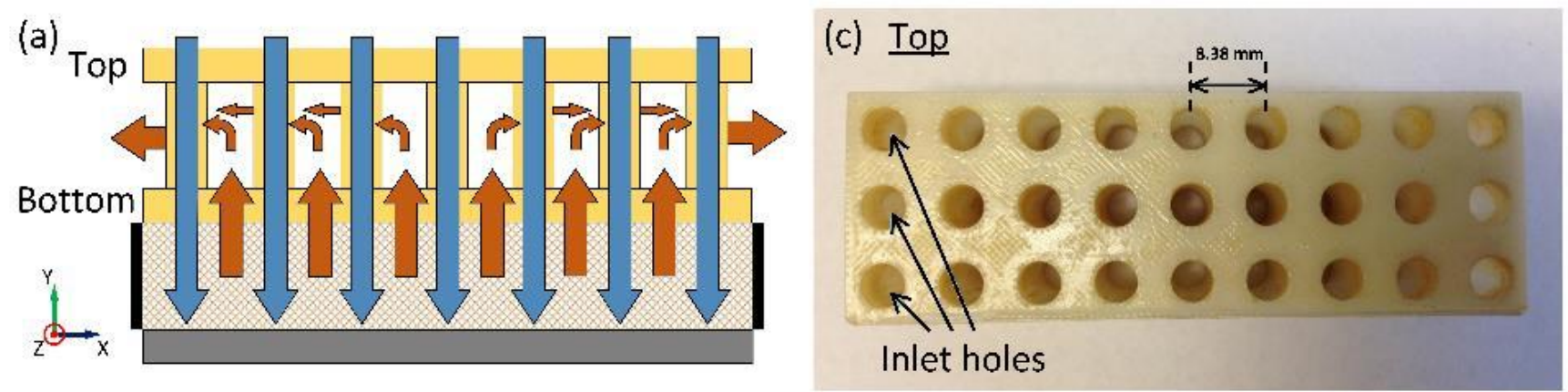

(b)

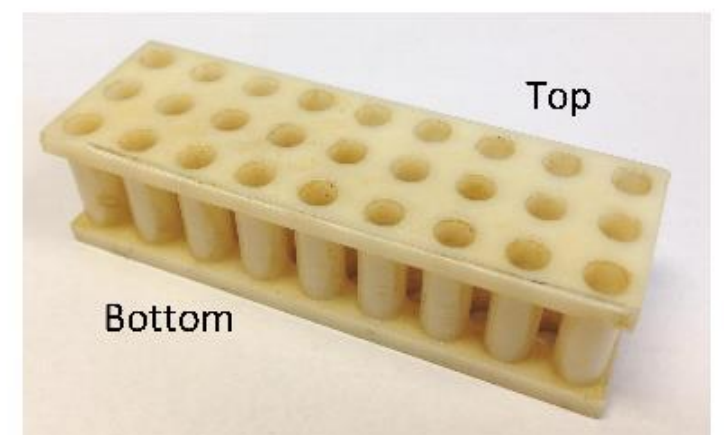

(d) Bottom

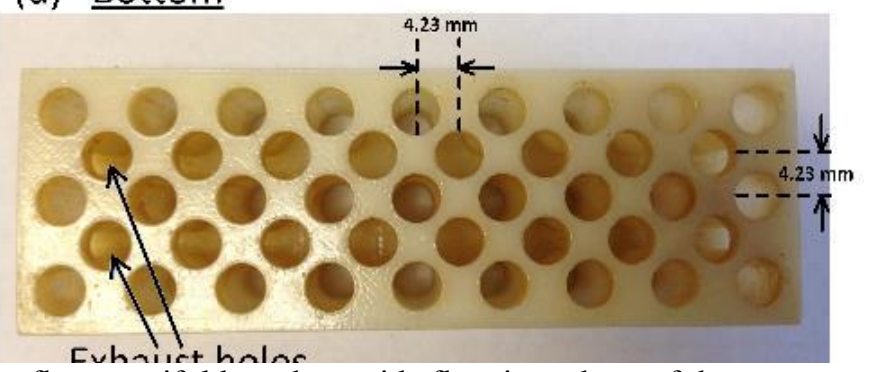

Fig. 2. (a) A schematic and (b) a picture of the 3D-printed tubular flow manifold used to guide flow in and out of the weaves. This flow manifold measures 76.2 × $22.2 \times 25.4 \mathrm{~mm}$. (c) The inlet area on the top surface with periodic channels that guide the impinging jet arrays across the manifold's full thickness and to the heated surface. (d) The outlet area on the bottom surface with periodic exhaust channels located between the inlet channels. These exhaust channels do not span the full manifold thickness so heated water flows between the top and bottom surfaces around the inlet channels and eventually leaves the manifold along the shorter edges on the left and right of the manifold as shown schematically in (a). 

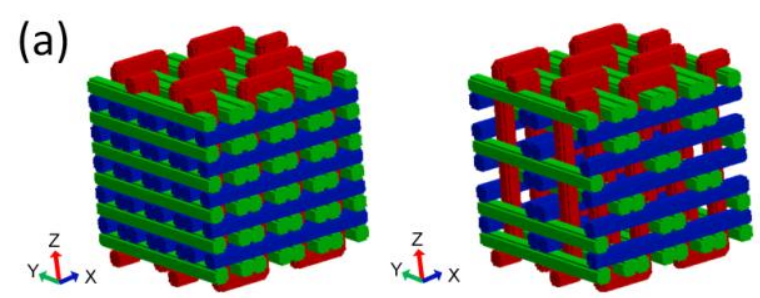

(b)

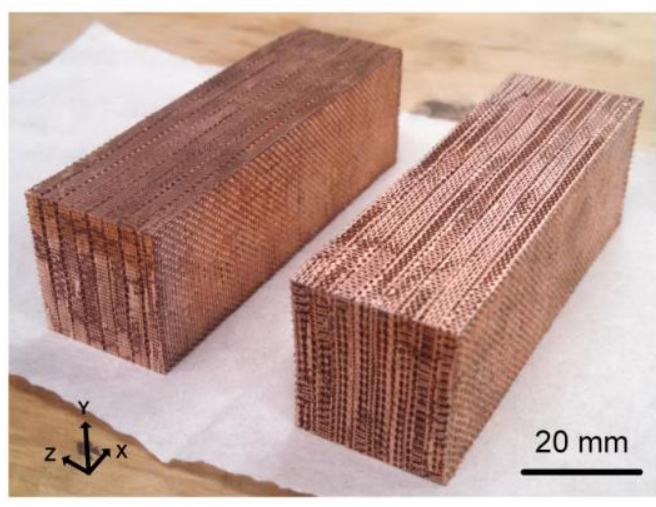

(c)

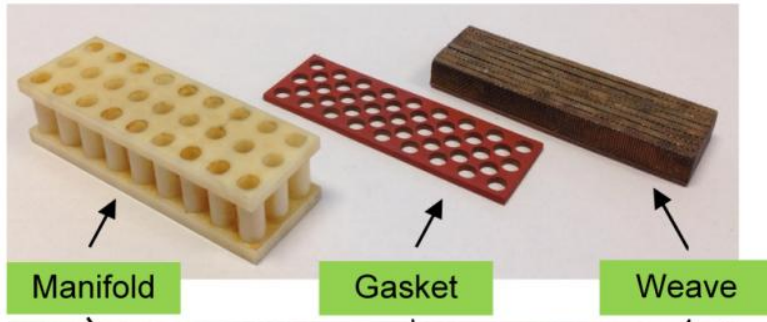

(d)

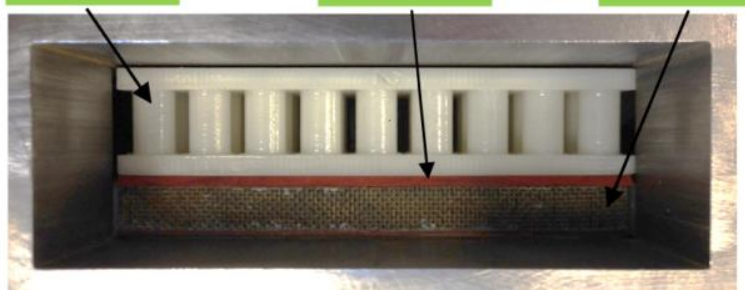

(e)

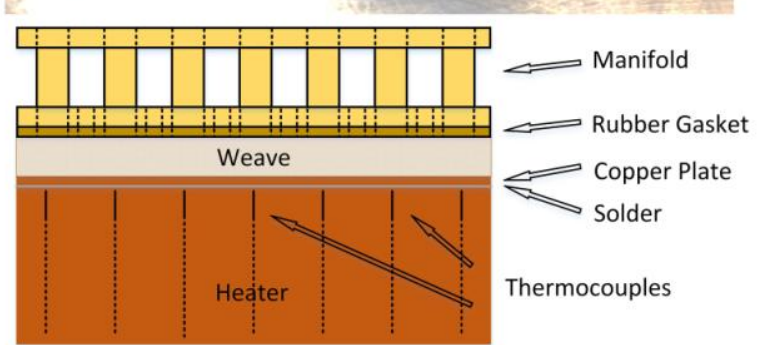

Fig. 3. (a) Schematic of woven "standard" and "optimized" architectures. (b) The standard and optimized Cu woven blocks measure 76.2 × $25.4 \times 23.4 \mathrm{~mm}$ before being cut to three different thicknesses ( $3.2 \mathrm{~mm}, 6.4 \mathrm{~mm}$ and $12.7 \mathrm{~mm}$ ) and being brazed to a $1 \mathrm{~mm}$ thick $\mathrm{Cu}$ face sheet. (c) An example of a combination of plastic manifold, rubber gasket and metallic weaves measures a total thickness of $25.4 \mathrm{~mm}$. (d) The full assembly in the test chamber. (e) The thermocouples are distributed evenly in the heater and are placed less than $1 \mathrm{~mm}$ from the bonding surface.

In addition to adjusting the thickness ratios between the weaves and manifolds to obtain an optimum solution, one can also vary other geometric features of the manifolds such as jet or inlet diameter, return or exhaust port diameter, spacing between inlet channels (labeled in Fig. 2c), spacing between inlet and exhaust channels (labeled in Fig. 2d), jet wall thickness, symmetry of the inlet/exhaust channel patterns, etc. These manifold features were held fixed to the values listed in Table 1, but will be varied in future studies. 
Table 1. The geometric features of the manufactured 3D-printed flow manifolds.

\begin{tabular}{|l|l|l|l|l|}
\hline Inlet channel & Exhaust channel & Jet wall & Top and bottom & Symmetry of the \\
diameter $(\mathrm{mm})$ & diameter $(\mathrm{mm})$ & thickness $(\mathrm{mm})$ & plate thickness \\
$(\mathrm{mm})$ & opening patterns \\
\hline 4.76 & 4.76 & 0.79 & 3.18 & Square \\
\hline
\end{tabular}

A schematic of the experimental setup is shown in Fig. 4. During each test, the cooling water flow rate was controlled using a regulation valve with an accuracy of $\pm 0.05 \%$, and its temperature at the inlet was $20 \pm 1{ }^{\circ} \mathrm{C}$. Pressure drops between the inlet and one of the two outlets were measured to an accuracy of $\pm 70 \mathrm{~Pa}$ using Omega HHP-803/SIL differential pressure transducers. Volumetric flow rate was measured with $\pm 1 \%$ accuracy using JLC International Inc. 100.21N IR-Opflow flowmeters. Three identical flowmeters were used, one for the inlet and the other two for the outlets. For heat flow, a transformer was used to maintain a constant heat flux $\left(20.7 \mathrm{~W} / \mathrm{cm}^{2}\right)$ into the system provided by ten cartridge heaters that were imbedded in a solid, $\mathrm{Cu}$ block. To measure the surface temperature, as depicted in Fig. 3e, seven evenly spaced type $\mathrm{T}$ thermocouples with an accuracy of $\pm 0.1^{\circ} \mathrm{C}$ after calibration were placed less than $1 \mathrm{~mm}$ from the bonding surface. The inlet and outlet temperatures of the coolant were measured using high precision Omega TH-44034 thermistors (accuracy: $\pm 0.1{ }^{\circ} \mathrm{C}$ ) located 254 $\mathrm{mm}$ away from the sample to obtain a ratio of section length to channel height of 10:1. Knowing the heat absorbed by the coolant from the measurements of the coolant temperature before and after heating, we quantified heat losses to the test fixture and surrounding environment to be between $8 \%$ and $12 \%$ by comparing the applied heat to the heat absorbed by the water. All the 
thermocouples were connected to a USB-Temp Data Acquisition device from Measurement Computing Inc. and were read by InstaCal and TracerDAQ software installed on a PC.

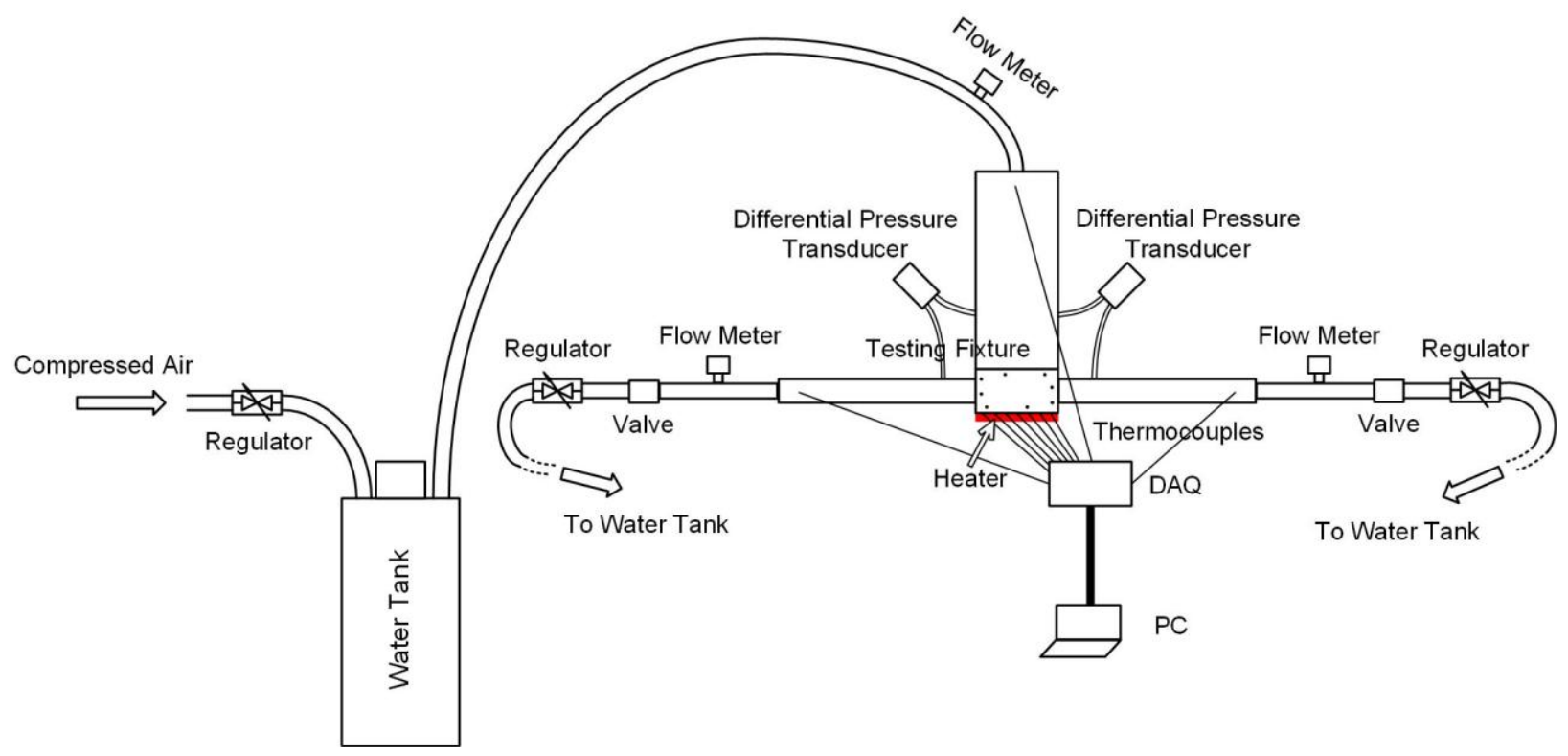

Fig. 4. Schematic of the testing setup.

To evaluate the performance of the manifolds by themselves, we replaced the weaves with the thin plastic frames shown in Fig. 5 that created open spaces between the manifold and the heating plate. The wall thickness of the $3 \mathrm{D}$ printed frames is $\sim 1 \mathrm{~mm}$ so they do not interfere with the water flow into or out of the two sets of channels within the manifolds. Similar experiments were then performed monitoring pressure drops, substrate temperatures and water temperatures.

With this experimental setup and procedure, the errors are produced due to limitations in the accuracies of experimental measurements and deviations of these measurements. Following ref [9], the propagation of individual uncertainties for pressure drop, average substrate 
temperature and maximum temperature non-uniformity are estimated to be $7 \%, 12 \%$ and $25 \%$, respectively.
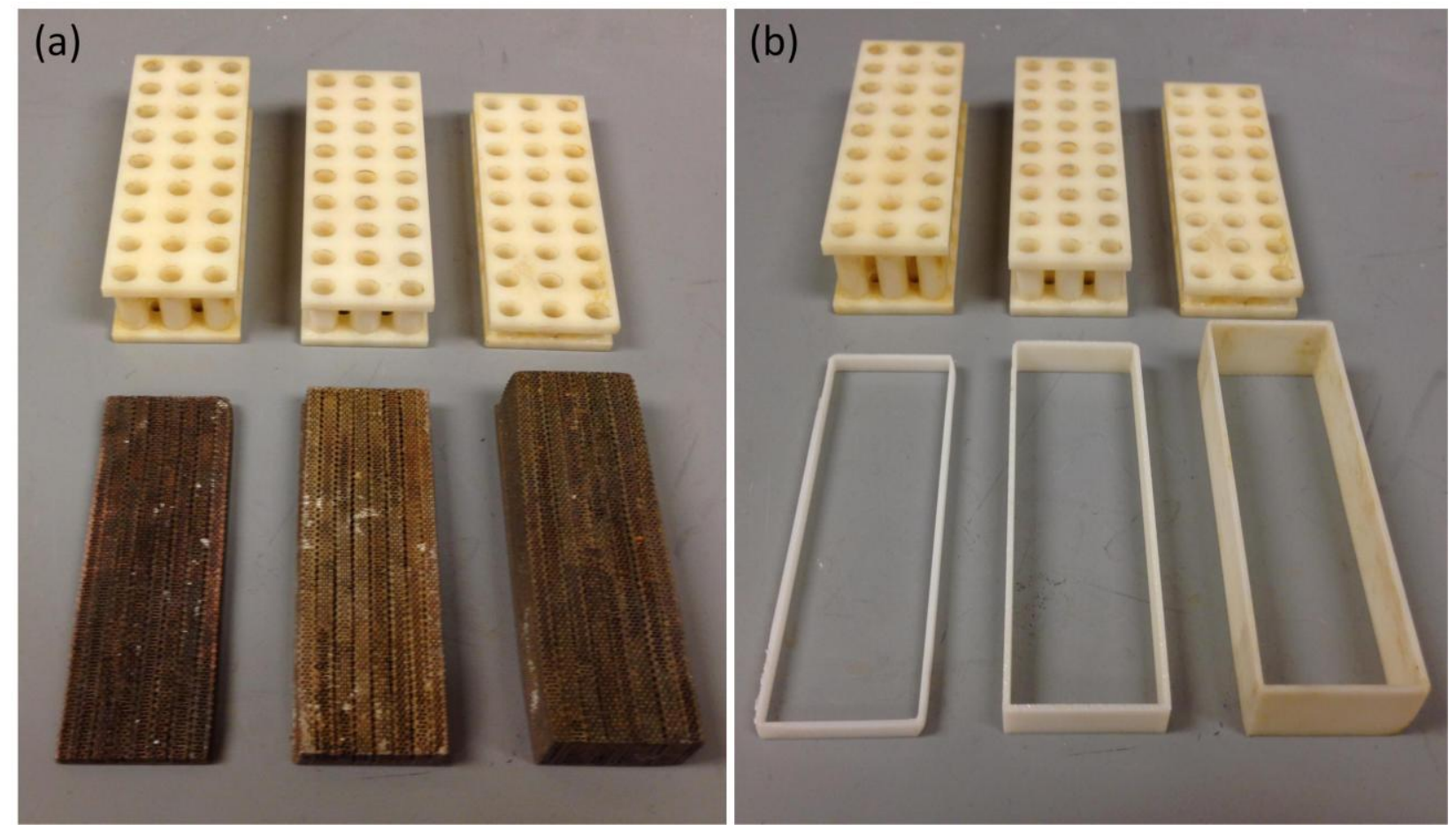

Fig. 5. (a) Three sets of manifolds and sliced weaves. (b) Three sets of manifolds and thin plastic frames designed to replace the weaves and create open spaces.

To explain the trends observed in the experiments, finite element modeling (FEM) was also performed (Fig. 6). The geometry is discretized with linear tetrahedral elements and the mesh size is refined based on surface curvature. The FEM is solved by iterating between the fluid and thermal models, solving temperature for an initial velocity and pressure field, and then updating and solving the velocity and pressure fields at this temperature. The iterations between the staggers continue until reaching a specified convergence tolerance. Due to the highresolution mesh that was required to capture the $202 \mu \mathrm{m}$ diameter wires and corresponding pore 
spaces, a reduced sample size was used in the $\mathrm{X}$ and $\mathrm{Z}$ directions by leveraging periodicity in the geometry and symmetry in the boundary conditions in these directions. In this study, the total number of elements are 0.67 to 6.04 million depend on the architecture and layers of weaves, and the average mesh size is $1.33 \times 10^{-4} \mathrm{~mm}^{3}$. We modeled a short X-direction length $(14.8 \mathrm{~mm})$ of the weave and manifold encompassing 0.75 inlets $(0.5+0.25)$ and 0.5 outlets near the outlet port and one full layer of weave thickness in the $\mathrm{Z}$ direction $(4.2 \mathrm{~mm})$, as shown in Fig. 6 . In the $\mathrm{Y}$ direction, the total thickness of the weave/open space and manifold was maintained at the full value of $25.4 \mathrm{~mm}$. We then segregated $25.4 \mathrm{~mm}$ into 8 units with each unit size being $3.2 \mathrm{~mm}$. As a result, for the three experimental thickness ratios studied here, the numbers of units for weaves/open spaces are 1,2 and 4, respectively, corresponding to the three thicknesses $(3.2 \mathrm{~mm}$, $6.4 \mathrm{~mm}$ and $12.7 \mathrm{~mm}$ ); while the numbers of units for manifolds are 7, 6 and 4, respectively, corresponding to the three thicknesses $(22.2 \mathrm{~mm}, 19.0 \mathrm{~mm}$ and $12.7 \mathrm{~mm})$. Therefore, the three thickness ratios between weaves/open spaces and manifolds are 1:7, 2:6 and 4:4. Fig. 6 demonstrates the case for the ratio being 4:4; thus the weave and manifold thicknesses are identical. In the simulations, we also studied other ratios ranging from 0.5:7.5 to 4:4 with an increment of 0.5 to achieve many data points. For the boundary conditions, no-slip conditions are assumed on all interfaces, the right side of the weave is assumed sealed by a no-slip wall, and the right side of the manifold perimeter serves as an outlet. The outlet is extended by a rectangular tube with the same cross-section area (not shown in the figure for clarity) and a length of 254 $\mathrm{mm}$ that matches the experimental setup. The reference pressure point is set at the far end of the extended tube to allow flow to develop. Thus, recirculation after the tube can be neglected. The other three sides have symmetric boundary conditions. From the expanded view in Fig. 6, more details about the wire geometries and bonding areas (yellow highlighted) are shown. The wires 
are assumed perfectly bonded across contacts except between $\mathrm{Z}$ wires and $\mathrm{X} / \mathrm{Y}$ wires, such that thermal conduction may occur across wire contact surfaces, and wire spacings are adapted from ref [7]. All the simulations were conducted using one flow rate $\left(3.1 \times 10^{-6} \mathrm{~m}^{3} / \mathrm{s}\right)$ and assuming laminar flow conditions, which we note is not the case in experiments. The focus here, however, is to study the differences between weaves and open spaces for various thickness ratios. Although results may vary at other flow rates, we believe that the study is useful for understanding system behavior and identifying qualitative trends. 


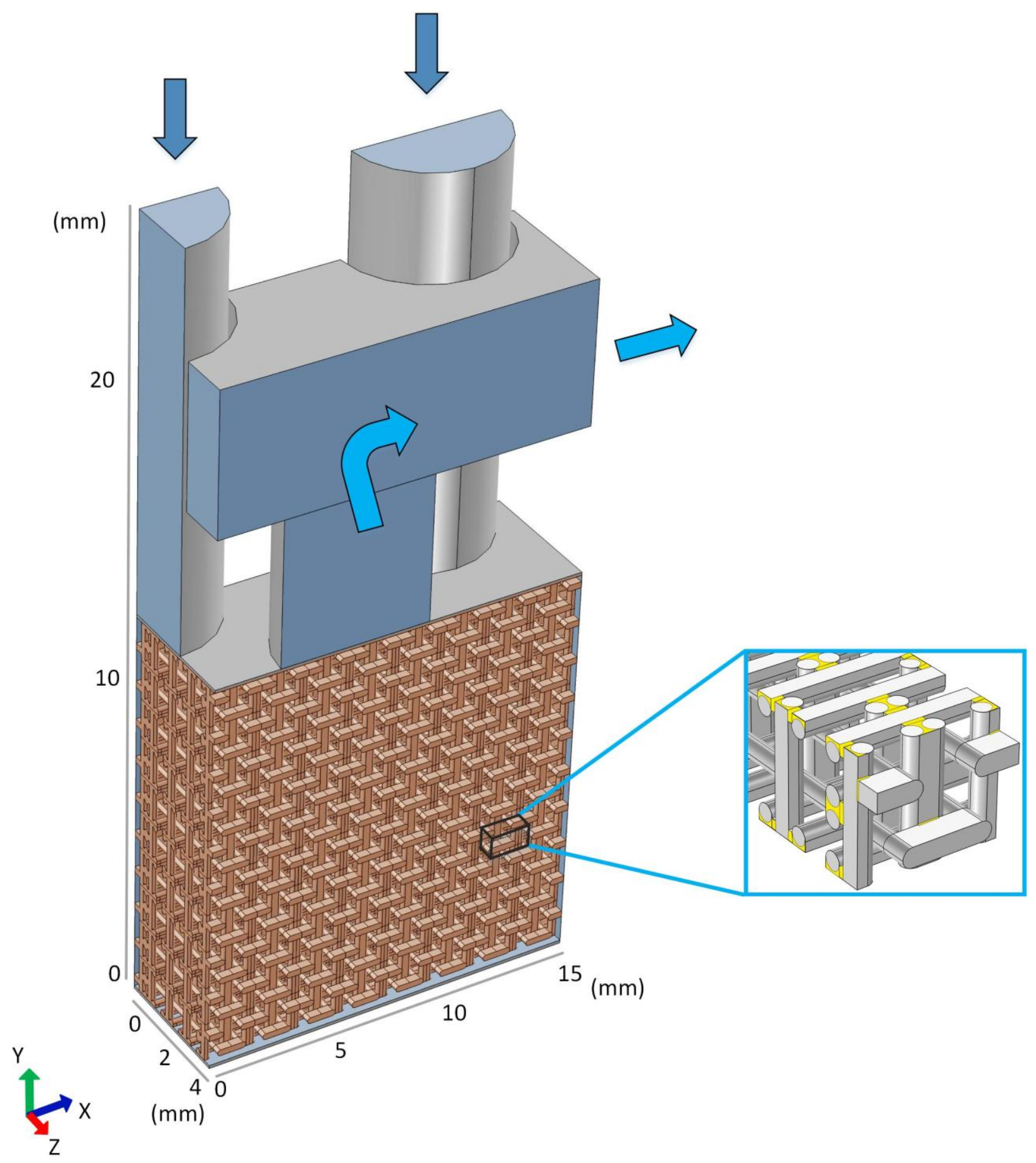

Fig. 6. Model of a reduced $14.8 \times 25.4 \times 4.2 \mathrm{~mm}$ specimen for finite element simulation. The thickness ratio between weaves/open spaces and manifolds varies from 0.5:7.5 to 4:4. This schematic shows an optimized weave combined with a flow manifold for a thickness ratio of 4:4. The expanded view shows the wire geometries and highlights the bonding contacts. The wires are assumed perfectly bonded contacts except between the $\mathrm{Z}$ and $\mathrm{X}$ or $\mathrm{Y}$ wires. Wire spacings are adapted from ref [7]. 


\section{Results and Discussions}

\subsection{Weave/manifold system vs. open space/manifold system}

The weave/manifold system (with standard and optimized weaves) was experimentally characterized and then compared to the open space/manifold system in Figs. 7, 9 and 11 using three performance indices:

- Pressure drop measured by the differential pressure transducer.

- Average surface temperature $T_{s}$ reported as the average of the seven thermocouples.

- Temperature variation across the surface $\Delta T$ reported as the difference between the maximum and minimum readings across the seven thermocouples.

For both systems, three different weave and open space thicknesses were combined with the manifolds of the appropriate thicknesses to create a $25.4 \mathrm{~mm}$ overall height. Finite element simulations were then performed on a model similar to Fig. 6 but using eight different thickness ratios including the three that were experimentally investigated. The corresponding simulation

results are shown in Figs. 8, 10 and 12, and predictions for the three experimentally investigated ratios are identified.

As the model idealization is quite different from experiments (idealized geometry without random flaws, sample length, flow rates, etc.), the simulations and experimental results cannot be directly compared. However, the simulations identify clear trends for each performance index. To quantify those trends we normalize the data by defining the lowest simulated value for each index as unity and present all other simulated values as a dimensionless ratio scaled to the lowest 
value. For pressure drop, the lowest value occurred in the open space/manifold system for an open space to manifold ratio of 1:7 (0.143) in Fig. 8. For the average surface temperature, the lowest value occurred in the standard weave/manifold system for a weave to manifold ratio of 0.5:7.5 (0.067) in Fig. 8. Lastly, for the temperature variation, the lowest value occurred on the standard weave/manifold system for a weave to manifold ratio of 4:4 (1) in Fig. 8. The lowest values are plotted with unit magnitude in Figs. 8, 10 and 12 and the other simulated values are scaled to these low points. The trends found within these simulations help to explain the experimental observations.

Figure 7 depicts pressure drops that were measured at different flow rates for each system. Several comparisons can be made. First, the weave/manifold system has $2 \mathrm{X}$ to $8 \mathrm{X}$ larger pressure drops than the open space/manifold system, due to the existence of the weave. Between the two different architectures of the weaves, the pressure drops on the standard weave are $20 \%$ to $35 \%$ higher due to its larger volume fraction of solid material and the fact that the wire patterns on the optimized weave were designed to achieve maximal flow rates $[7,10,11]$. Second, pressure drop is the lowest at the intermediate weave thickness $(6.4 \mathrm{~mm}$ weave + manifold $)$ for the weave/manifold system, regardless of the weave architecture, pressure drop rises with the open space thickness for the open space/manifold system. These two trends are attributed to the fact that the total pressure drop is an addition of two sub-pressure drops. One occurs across the weave or open space and the other occurs across the manifold, and the two have opposite dependencies on the thickness ratios. This phenomenon can be examined more clearly by the simulation results in Fig. 8. In Fig. 8 it can be seen that when the weave is thicker and the manifold is thinner (larger thickness ratio), the pressure drop decreases on the weave while it increases on the manifold. In addition, pressure drop increases significantly when the thickness 
of either the weave or manifold becomes very small. Thus, the lowest total pressure drop is predicted to occur at an intermediate thickness ratio. The experimental results support this prediction as the intermediate weave/manifold system $(6.4 \mathrm{~mm}$ weave + manifold $)$ shows the lowest total pressure drop. A similar minimum is predicted and observed for open space/manifold system, but because the pressure drop on the open space is much smaller compared to that on the manifold the minimum occurs at a small thickness ratio which corresponds to the $3.2 \mathrm{~mm}$ open space + manifold system.

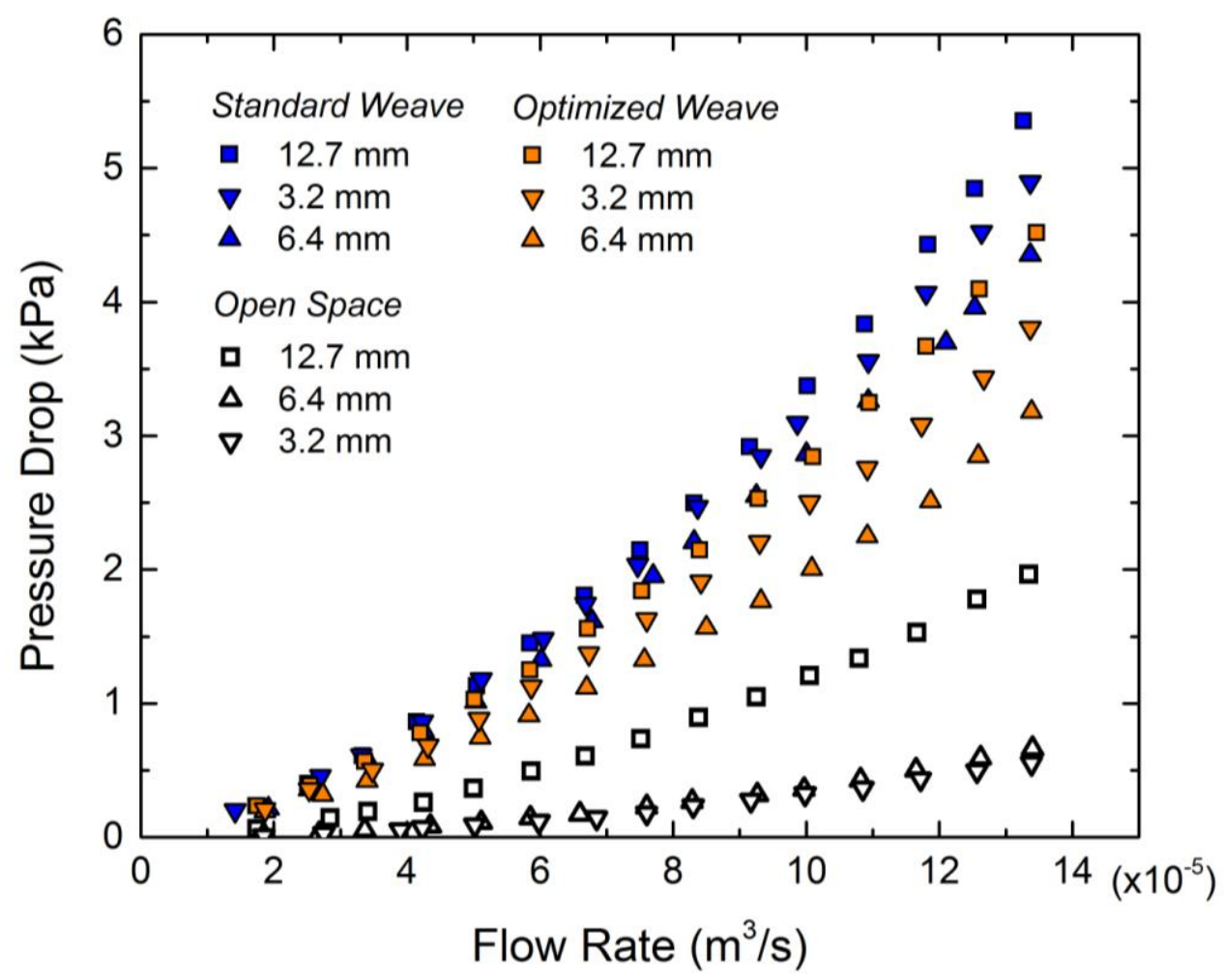

Fig. 7. Pressure drop measurements for combinations of manifolds with either weaves or open spaces. The overall thickness is $25.4 \mathrm{~mm}$ and weaves or open spaces have thicknesses of $12.7 \mathrm{~mm}, 6.4 \mathrm{~mm}$ or $3.2 \mathrm{~mm}$. 


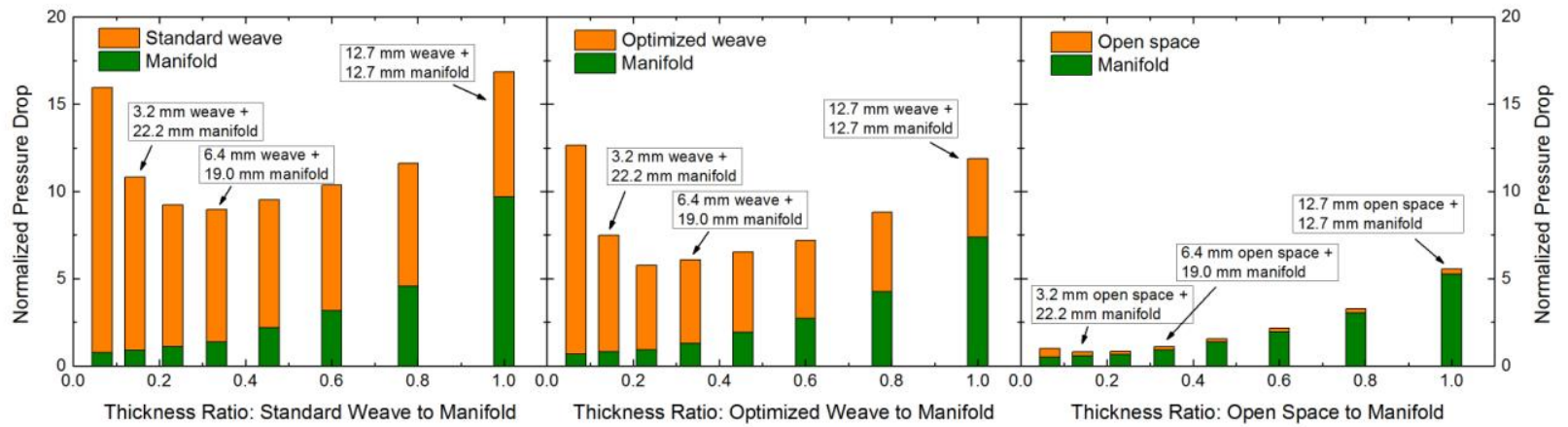

Fig. 8. Scaled pressure drop simulations for combinations of manifolds with either weaves or open spaces. The total thickness is maintained at 8 unit lengths while the thickness ratios between the weaves or open spaces and the manifolds vary from 0.5:7.5 to 4:4. The pressure drop is normalized using the minimum simulated value, which appeared for the open space/manifold system with a thickness ratio of 1:7 (0.143). The three experimentally characterized ratios are identified.

For the heat transfer study the average surface temperature $T_{s}$ and the temperature variation across the surface $\Delta T$ depend on the inserted material (weave or open space) and the flow rates. Figs. 9-10 and Figs. 11-12 present the measured and simulated values for $T_{S}$ and $\Delta T$, respectively. 


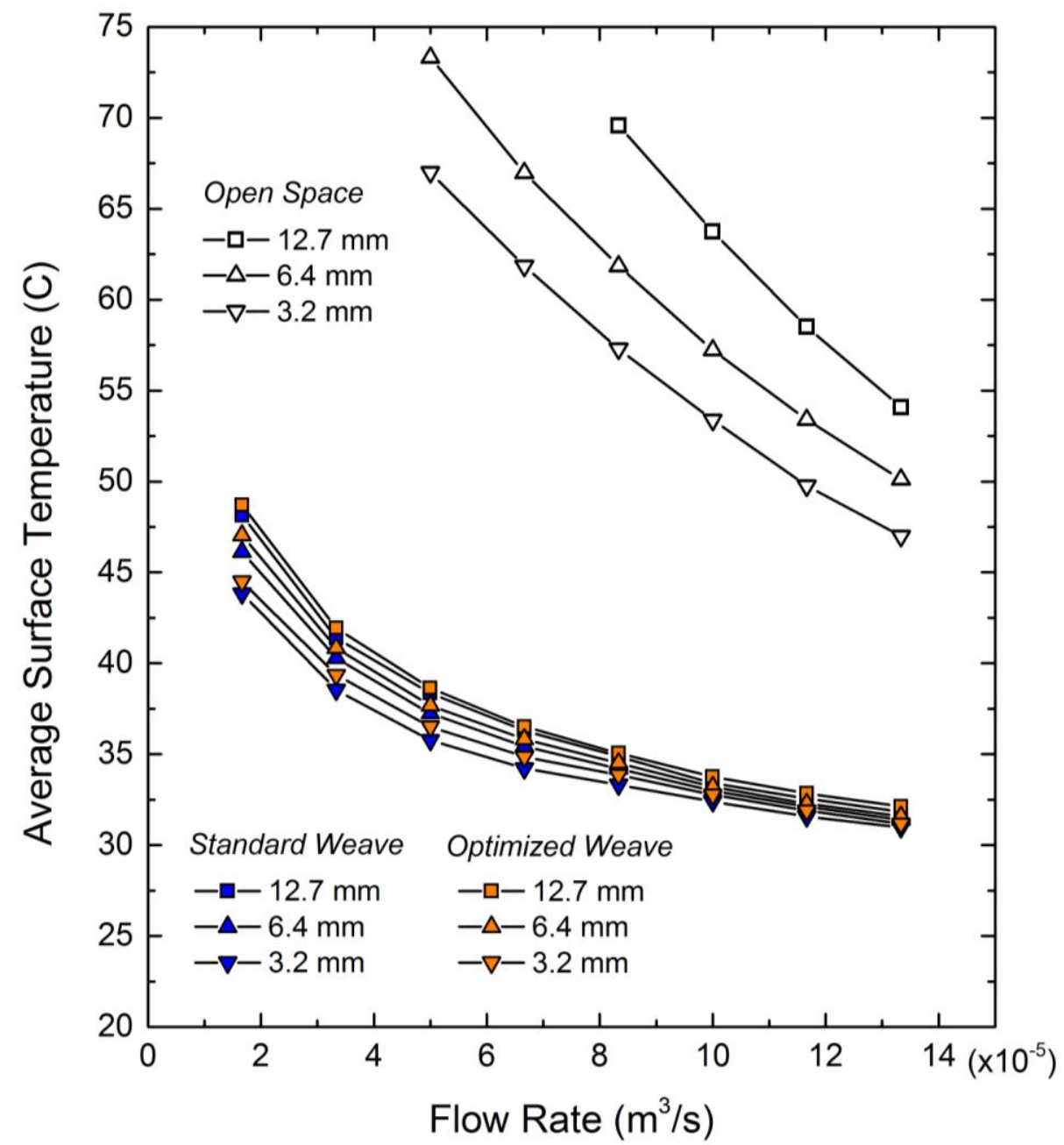

Fig. 9. Measured average surface temperatures for combinations of manifolds with either weaves or open spaces. The overall thickness is $25.4 \mathrm{~mm}$ and the thicknesses of the weaves or open spaces are either $12.7 \mathrm{~mm}, 6.4 \mathrm{~mm}$ or $3.2 \mathrm{~mm}$. 


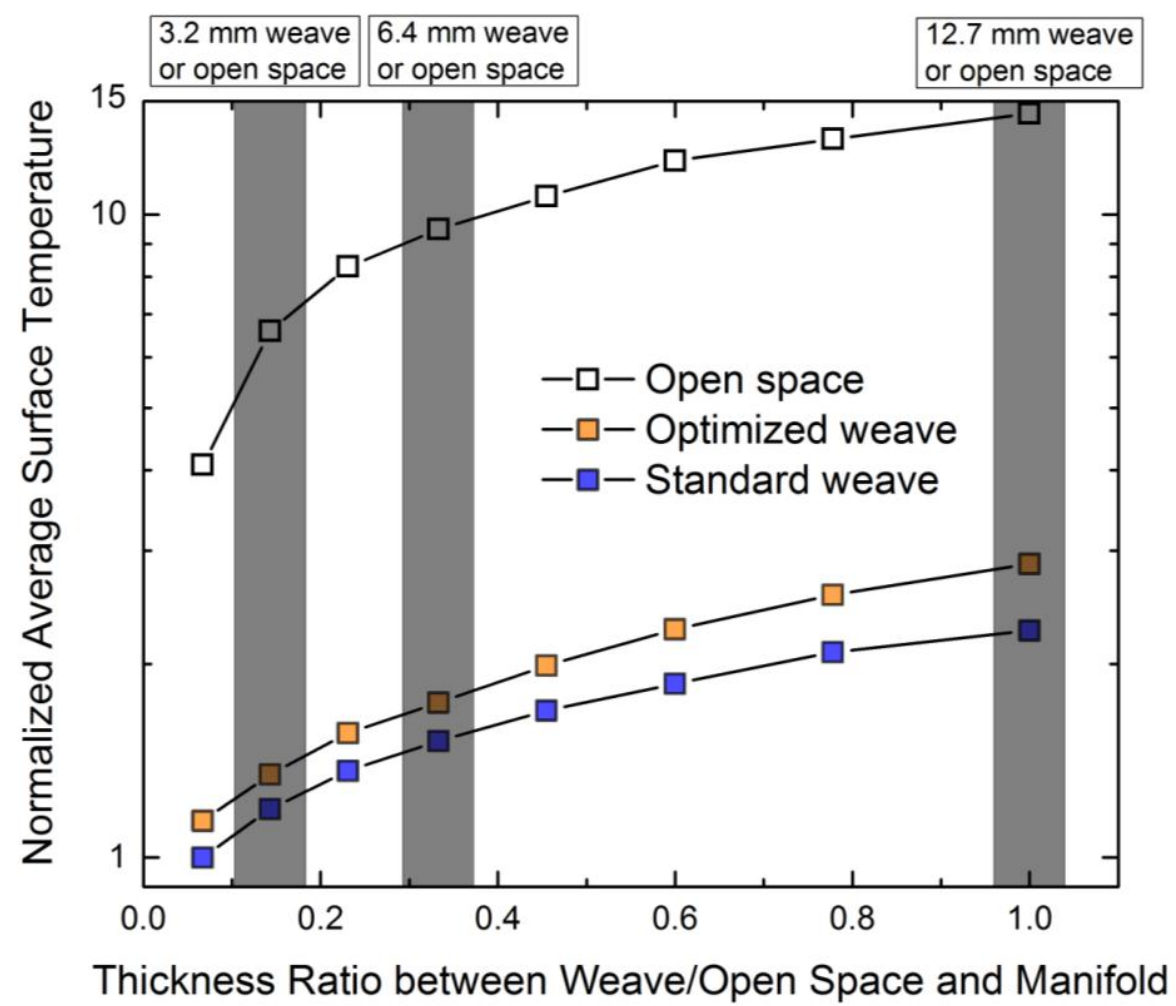

Fig. 10. Average simulated surface temperatures for combinations of manifolds and either weaves or open spaces. The total thickness is maintained at 8 unit lengths while the thickness ratios between the weaves or open spaces and the manifolds vary from $0.5: 7.5$ to $4: 4$. The average surface temperature is normalized by the minimum value, which appeared for the standard weave/manifold system with a thickness ratio of 0.5:7.5 (0.067). The three experimentally characterized ratios are identified. 


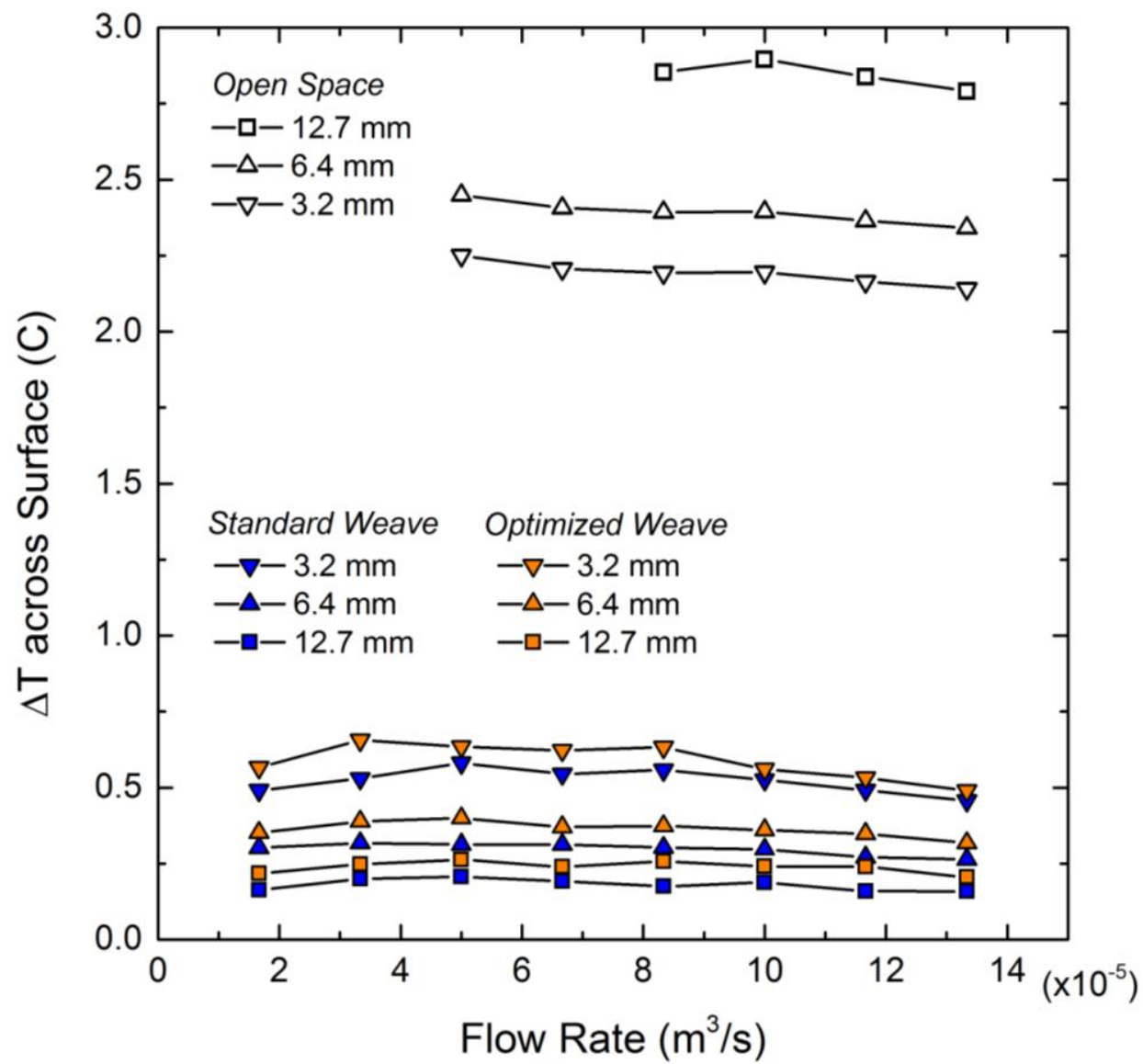

Fig. 11. Temperature variation measurements for combinations of manifolds and either weaves or open spaces. The overall thickness is $25.4 \mathrm{~mm}$ and in three circumstances the thicknesses of weaves or open spaces are separately $12.7 \mathrm{~mm}, 6.4 \mathrm{~mm}$ and $3.2 \mathrm{~mm}$. 


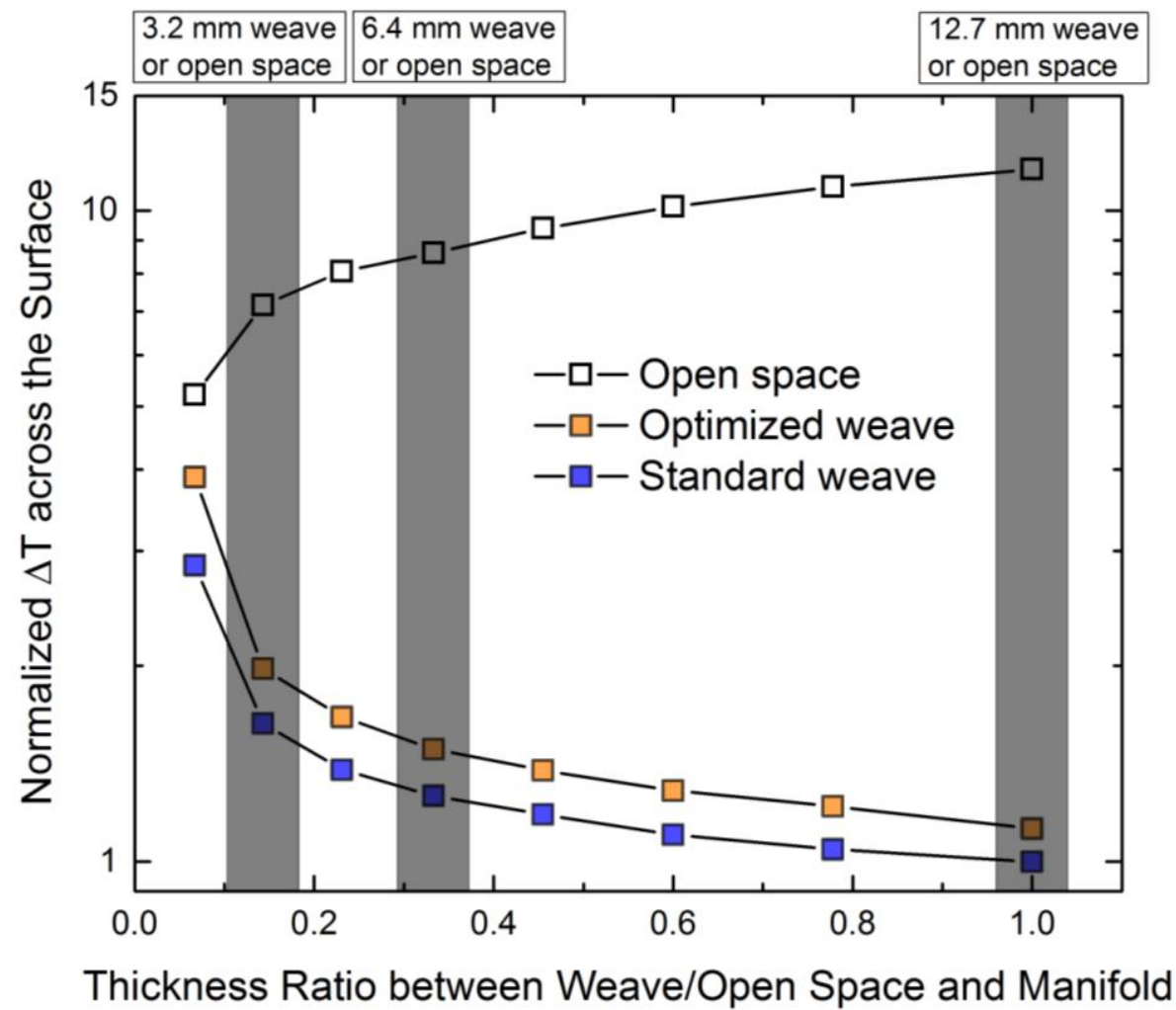

Fig. 12. Simulated temperature variations for combinations of manifolds with either weaves or open spaces. The total thickness is maintained at 8 unit lengths while the thickness ratios between the weaves or open spaces and the manifolds vary from 0.5:7.5 to 4:4. The temperature variation is normalized by the minimum simulated value, which appeared for the standard weave/manifold system with a thickness ratio of 4:4 (1). The three experimentally characterized ratios are identified.

Several trends can be observed in the experimental data. First, the average surface temperatures $T_{S}$ and temperature variations $\Delta T$ are significantly lower (2-3 times and 5-15 times, respectively) when the manifold is combined with a weave rather than an open space. The weaves fill much of the space with solid wires that conduct heat throughout the space and provide more surface area for enhancing thermal convection at a given flow rate. This can also be observed with the simulation data in Figs. 10 and 12. With the absence of the heat exchange 
media, $T_{S}$ and $\Delta T$ rise by up to $6 \mathrm{X}$ and $10 \mathrm{X}$, respectively, when comparing the weave/manifold system to the open space/manifold system.

Second, from Figs. 9 and 10 it can be seen that $T_{S}$ increases as the ratio of the weave/open space thickness to the manifold thickness rises, regardless of whether a weave or an open space is present. We attribute this performance to two factors that compete with each other for the weave/manifold combination: (1) the volume of wires that are available to conduct and convect heat, and (2) the proximity of the streamlines to the substrate. When the weave is thick, more wires are available to enhance thermal conduction and convection, but less coolant travel through the full weave to reach the surface of the substrate. Conversely, when the weave is thin, fewer wires are available to enhance thermal conduction and convection, but more coolant reaches the surface of the substrate. The experimental data strongly suggests that the second factor dominates in these experiments and in fact a thinner weave is preferred. Simulations support this conclusion and show that a temperature gradient builds up at the bottom of the thick weaves which eventually increases $T_{s}$. For the open space/manifold systems that lack a weave, thinner open spaces allow more direct impingement of the coolant on the substrate and hence show the best performance.

Third, from Figs. 11 and 12 it can be seen that $\Delta T$ decreases as the ratio of the weave thickness to the manifold thickness rises but $\Delta T$ increases as the ratio of the open space thickness to the manifold thickness rises. For the weave/manifold system, thicker weaves smooth the temperature more effectively as more wires enhance thermal conduction and lessen flow disturbance close to the substrate. In the case of open spaces though, without the wires distributing the heat horizontally, the larger $\Delta T$ for thicker open spaces is attributed to the overall higher $T_{S}$ on the substrate. 
Fourth, in Fig. 11 a wavy pattern of $\Delta T$ was observed at different flow rates for most of the weave/manifold systems. The wavy pattern is attributed to the fact that the magnitude of $\Delta T$ is determined by the combination of two factors: thermal conduction within the solid wires and thermal convection between wires and water. When flow rate is low, thermal conduction in the wires prevails, causing an overall smoothing of the surface temperature and a lowering of $\Delta T$. In contrast, when flow rate is high, thermal convection prevails, and lowers the surface temperatures in general. As a result, $\Delta T$ is lower when flow rate is either low or high, causing a wavy pattern over different flow rates. This phenomenon was also observed in our previous study [1].

Lastly, within the weave/manifold system, $T_{S}$ and $\Delta T$ are slightly lower (less than $20 \%$ ) when using the standard architecture than the optimized architecture for the same thickness ratio. This is simply due to the higher wire density in the standard architecture and the resulting increased ability to conduct and convect heat.

\subsection{Weave/manifold distributed array vs. axial and bifurcated flow patterns}

We then compare the new weave/manifold system as shown in Fig. 2 with the previously studied weave only system to identify improvements gained through the addition of the manifold. Two flow patterns were studied on the weave only system: axial and bifurcated (called 'full bifurcated' in [1]), as illustrated in Fig. 1. The new weave/manifold system here provides a more complicated 3D flow pattern which we call a distributed array. For the axial, bifurcated and distributed array flow patterns we maintain the same heat flux $\left(20.7 \mathrm{~W} / \mathrm{cm}^{2}\right)$ to the heater block and the total thickness of the system is $25.4 \mathrm{~mm}$. For the axial or bifurcated flow pattern, the full 
$25.4 \mathrm{~mm}$ thick woven block was used for testing, whereas for the distributed array flow pattern, the intermediate $6.4 \mathrm{~mm}$ thick woven block $+19.0 \mathrm{~mm}$ thick manifold combination was used for the simplicity of comparison. In addition, only the optimized architecture is compared here but the standard architecture was shown to offer the same trends. Comparisons of pressure drops, average surface temperatures and temperature variations are plotted in Figs. 13-15 and several points standout.

First, the highest, intermediate ( $\sim \mathrm{X}$ lower) and the lowest $(\sim 15 \mathrm{X}$ lower) pressure drops occur under axial, distributed array, and bifurcated flow, respectively. The highest pressure drops that are seen for the axial flow are attributed to this system's much longer flow paths in the weave, while the lowest pressure drops record for the bifurcated flow are attributed to this system's short flow paths due to edge effects. The distributed array has a relatively short flow path within the weave as well, but the 180 degrees turn from the inlet to outlet channels increases the resistance to flow and hence raises the pressure drop across the system. However, note that the pressure drop for the distributed array system only exceeds that of the bifurcated flow slightly and thus would not increase pumping requirements significantly. 


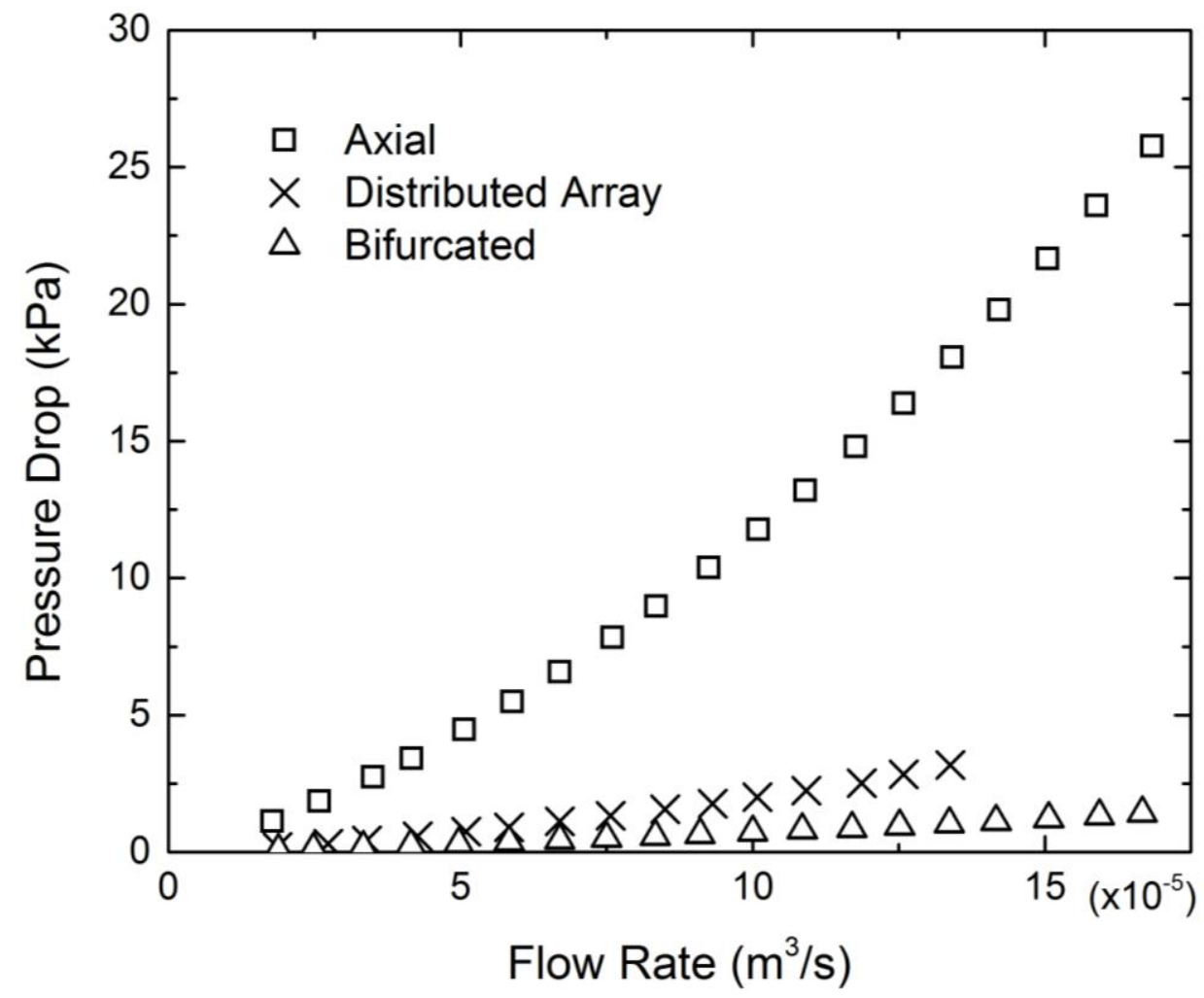

Fig. 13. Comparison of pressure drop vs. flow rate for three flow patterns: axial, bifurcated and weave/manifold distributed array. The axial and bifurcated flow patterns were performed on $25.4 \mathrm{~mm}$ thick optimized woven blocks, while the distributed array flow pattern was performed on the combination of a $6.4 \mathrm{~mm}$ thick optimized woven block and a $19.0 \mathrm{~mm}$ thick flow manifold.

Second, as shown in Fig. 14, the average surface temperatures in the distributed array flow case are reduced by approximately 50\% compared to the bifurcated flow case and $20 \%$ higher than the axial flow case. This was viewed as excellent performance given that the distributed array flow system uses $75 \%$ less conductive material $(\mathrm{Cu})$ than the other cases. Although the axial flow still possesses the advantage of having the cooling water sweeping past the heated surface, this water is heated as it flows along the length and thus loses cooling effectiveness as the length of the specimen increases. The distributed array, on the other hand, allows cooling water to enter and heated water to exit at many points along the weave and is thus 
highly scalable. Although the bifurcated flow case also allows cooling water to enter along the length, heated water remains in the system longer, also degrading effectiveness. It can also be noted that the average surface temperature for the distributed array flow case drops slightly faster than that for the axial flow case with increasing flow rates, bringing the temperatures for both cases closer at high flow rates. This is due to the fact that while the streamlines of the axial flow vary little at different flow rates, the streamlines of the distributed array tend to reach deeper and thus closer to the heated surface at higher flow rates compared to low flow rates. This shift enhances heat transfer.

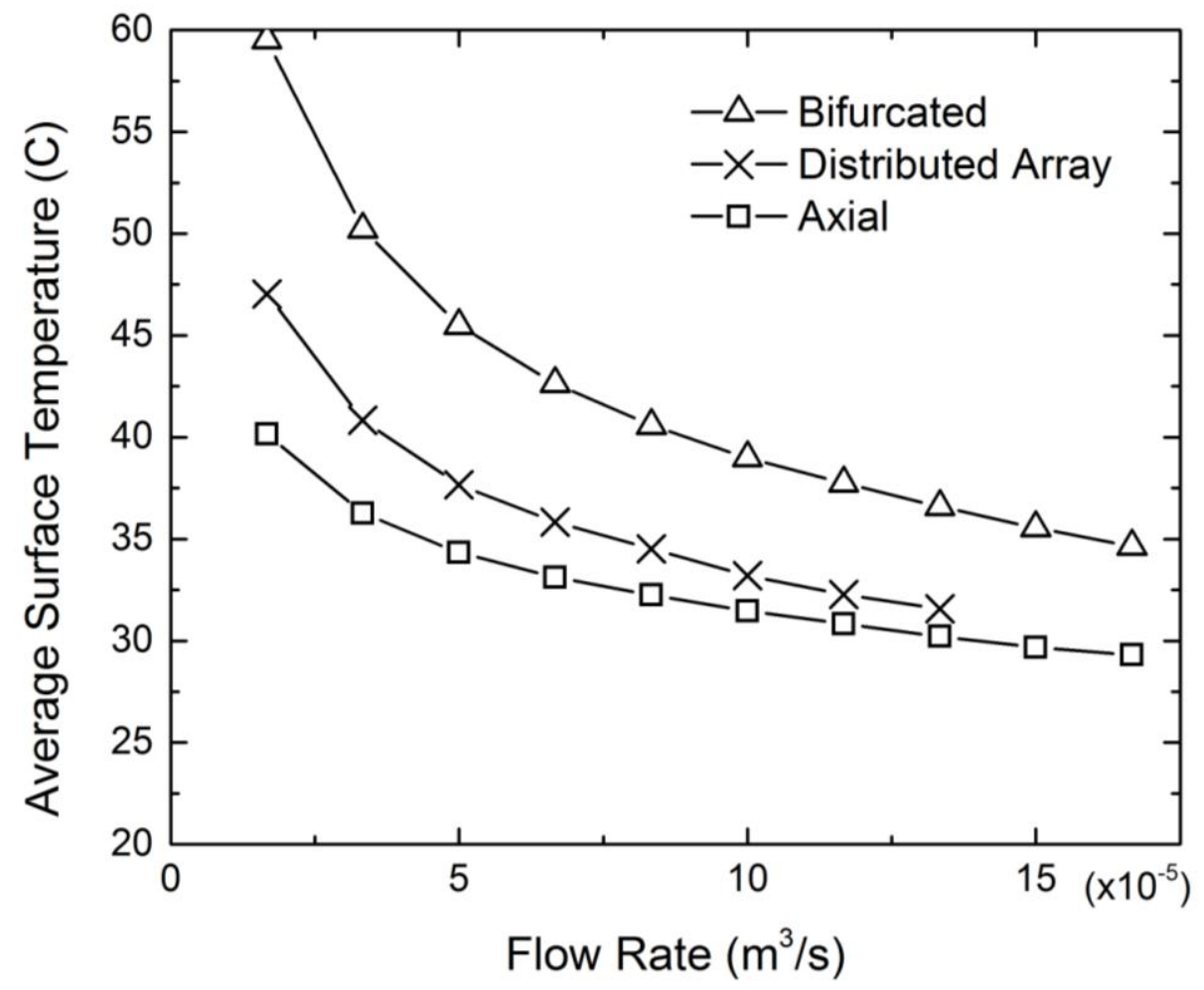

Fig. 14. Comparison of average surface temperature vs. flow rate for three flow patterns: axial, bifurcated and weave/manifold distributed array. The axial and bifurcated flow patterns were performed on $25.4 \mathrm{~mm}$ thick optimized woven blocks, while the distributed array flow pattern was performed on the combination of a $6.4 \mathrm{~mm}$ thick optimized woven block and a $19.0 \mathrm{~mm}$ thick flow manifold. 
Lastly, as shown in Fig. 15, $\Delta T$ across the surface is the lowest in the distributed array flow pattern, compared to the axial flow pattern (9-15 times higher) or the bifurcated flow pattern (4 times higher). The low $\Delta T$ of the weave/manifold system can be attributed to the fact that the manifold injects the coolant uniformly over the heated surface and also allows heated fluid to exit uniformly. The patterns of the inlet and outlet channels allow the full surface area to be segmented into many unit cells, which dramatically increases temperature uniformity. Once the properties in one unit cell are optimized, they can be distributed across a large surface area by merely repeating the unit cell pattern within the manifold. For the axial or the bifurcated flow patterns, though, the temperature variations scale with the size of the sample. This is a particularly important distinction when cooling large heated areas.

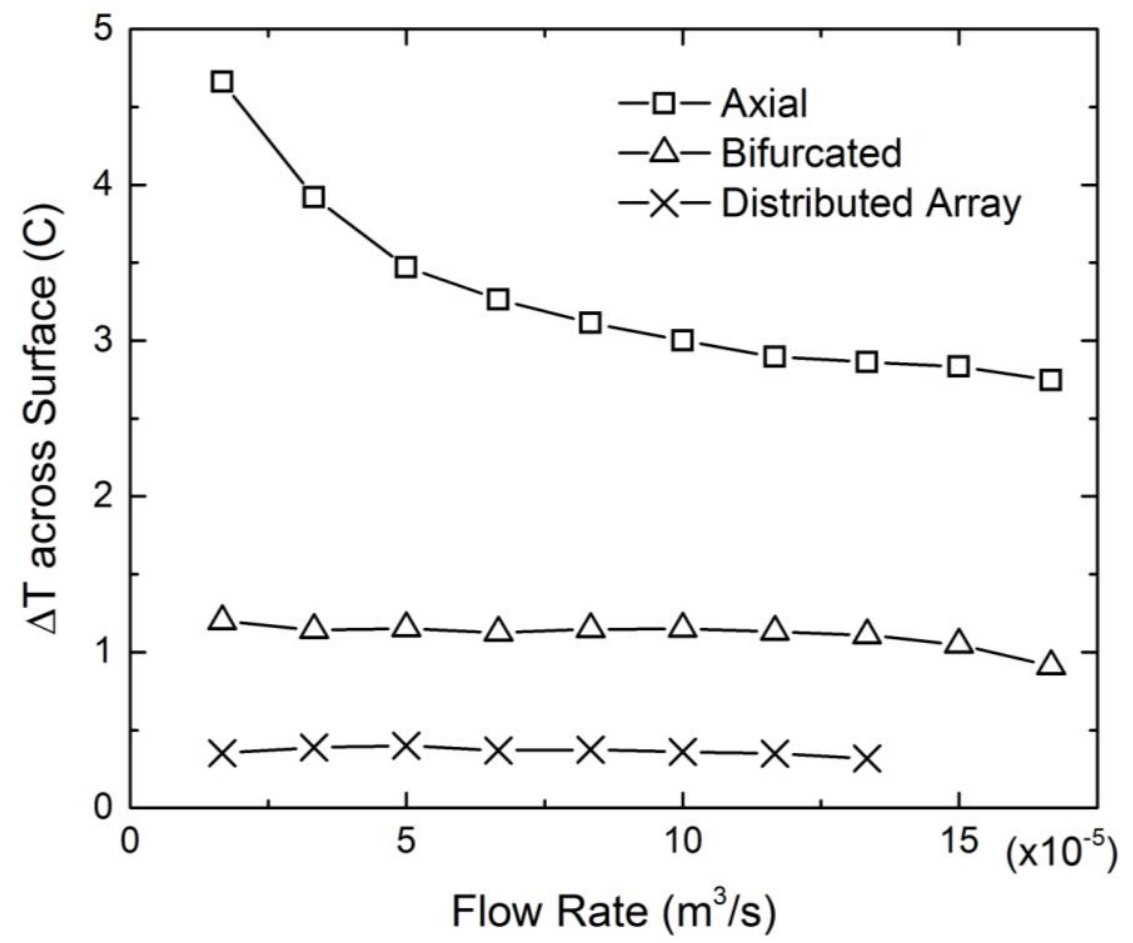


Fig. 15. Comparison of $\Delta T$ across the surface vs. flow rate for three flow patterns: axial, bifurcated and weave/manifold distributed array. The axial and bifurcated flow patterns were performed on $25.4 \mathrm{~mm}$ thick optimized woven blocks, while the distributed array flow pattern was performed on the combination of a $6.4 \mathrm{~mm}$ thick optimized woven block and a $19.0 \mathrm{~mm}$ thick flow manifold.

\subsection{Thermal performance vs. pumping power}

The design of an effective heat exchanger requires high thermal performance (i.e. low average surface temperature $T_{S}$ and low temperature variation $\Delta T$ ) and low mechanical pumping power to drive the fluid through the heat exchanger. Pumping power is a product of the pressure drop $(\Delta \mathrm{P})$ and the volumetric flow rate, indicating the energy needed per second to overcome fluid friction within the heat exchanging system. Below we have calculated $T_{S}$ and $\Delta T$ at different pumping powers and compare the weave/manifold with the open space/manifold system (Fig. 16), as well as the three flow patterns mentioned above (Fig. 17).
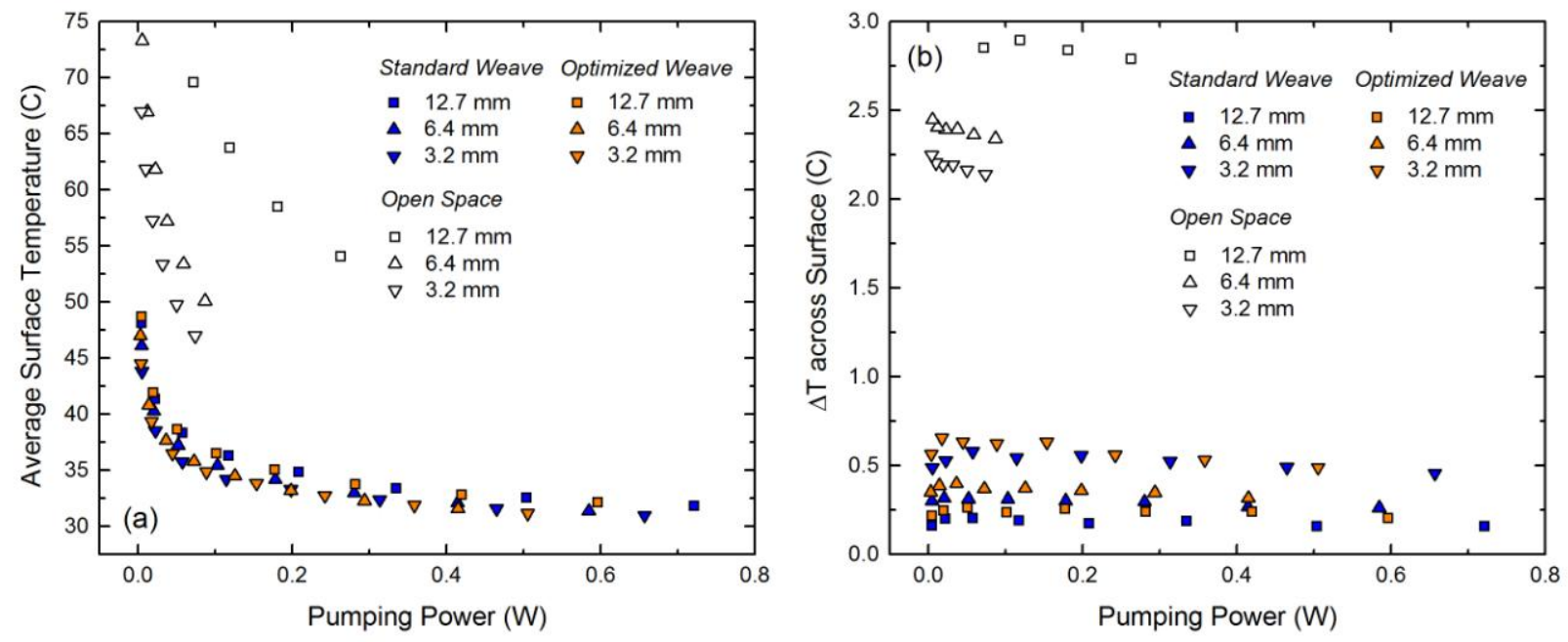
Fig. 16. Comparison of (a) average surface temperature $T_{S}$ and (b) temperature variation $\Delta T$ at different pumping powers for combinations of manifolds with either weaves or open spaces. The overall thickness of the exchange system is $25.4 \mathrm{~mm}$ and the weaves or open spaces have thicknesses of $12.7 \mathrm{~mm}, 6.4 \mathrm{~mm}$ or $3.2 \mathrm{~mm}$.
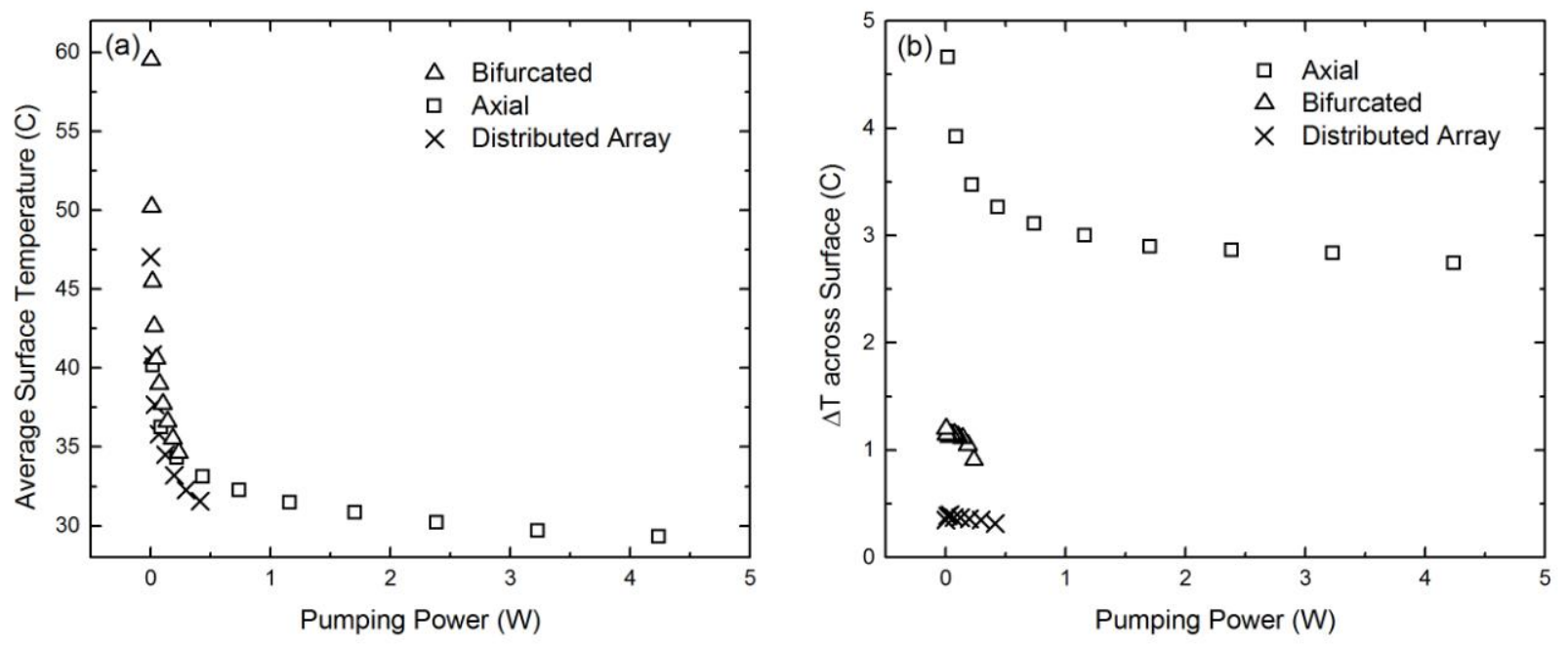

Fig. 17. Comparison of (a) average surface temperature $T_{s}$ and (b) temperature variation $\Delta T$ at different pumping powers for three flow patterns: axial, bifurcated and weave/manifold distributed array. The axial and bifurcated flow patterns were performed on $25.4 \mathrm{~mm}$ thick woven blocks, while the distributed array flow pattern was performed on the combination of a $6.4 \mathrm{~mm}$ thick optimized woven block and a $19.0 \mathrm{~mm}$ thick flow manifold.

Several points can be raised when comparing the weave/manifold system to the open space/manifold system in Fig. 16. First, the average surface temperatures $\left(T_{s}\right)$ are up to 3 times lower and the temperature variations $(\Delta T)$ are up to 15 times lower than those for the open space/manifold systems at the same pumping power. This demonstrates that even after accounting for the low pressure drops in the open space/manifold system, the weave/manifold system still possesses the better thermal performance. Second, in the weave/manifold system there is no obvious difference between the two architectures, in both $T_{s}$ and $\Delta T$, at a given pumping power. The slightly lower $T_{s}$ and $\Delta T$ values observed earlier in the standard architecture 
for a given flow rate, are overcome by a higher $\Delta P$ for this denser architecture. Third, between the three thicknesses of weaves, the thicker weaves still possess a slightly higher $T_{S}$ and a lower $\Delta T$ at a given pumping power.

Turning to the thermal performance of the axial, bifurcated and weave/manifold distributed array flow patterns as a function of pumping power, similar changes are observed in Fig. 17. The distributed array now has the lowest $T_{s}$ at a given pumping power (Fig. 17a), due to its lower $\triangle P$ than the axial flow and its lower $T_{s}$ than the bifurcated flow. However, the differences in surface temperatures are not significant at a given pumping power. For temperature uniformity (Fig. 17b), though, the earlier relationship still exists between the three flow patterns, with axial flow having the highest variations in temperature and the weave/manifold system having the lowest. The $\Delta T$ values are up to $10 \mathrm{x}$ lower for the weave/manifold system at low pumping powers. Taken together, Fig. 17 suggests that when pumping power is limited, the weave/manifold distributed array system is the superior choice, offering better thermal performance than the axial or bifurcated flow systems, particularly at low pumping powers.

\subsection{Optimization of the manifold}

While the weave/manifold system has shown significant improvements compared to either the open space/manifold system or the weave only system, the designs of the manifold or the heat exchanger media (weave in this study) have not yet been fully optimized. One could utilize topology or shape optimization to either optimize the geometric parameters of the current system or create a new design combining the manifold and weave as one piece. With the help of 3D 
printing techniques, many novel designs can now be realized despite their complex shapes. This may enable the design of more effective heat exchanger systems that address the ever-increasing thermal load requirements of high power electronic devices and other heat producing systems.

A simple example of adjusting the geometric parameters can be shown in Fig. 18a, where the outlet hole diameter is reduced to only $62.5 \%$ of the inlet hole diameter. As the total exhaust area reduces, the flow velocity in the weave is expected to increase, the streamlines in the weave are expected to reach deeper, and this should result in higher pressure drops and lower average surface temperatures. To compare this new manifold design with the original design, we chose the thickest optimized weave to accentuate differences in penetration, and the corresponding pressure drops and average surface temperatures are shown in Fig. 18b. It is clear that with the new manifold, one property improves (average surface temperature) while another property deteriorates (pressure drop). This implies that one can optimize the properties of the system to satisfy the requirements of different applications.

Finally, we note that the manifold in this study was 3D printed in ABS to limit thermal conduction and minimize heat transfer through the manifold, allowing the study to focus on flow patterns and woven materials. However, the manifold itself could also be made of conductive material (such as $\mathrm{Cu}$ ) and contribute to heat transfer within the system. This would essentially allow heat transfer to begin at the top of the manifold and lead to temperature rises in the cooling water before it enters the woven material. In this case, we found that although mass (and cost) increased as expected, pressure drop remained the same, average substrate temperature decreased, and substrate temperature uniformity remained approximately the same. 
(a)

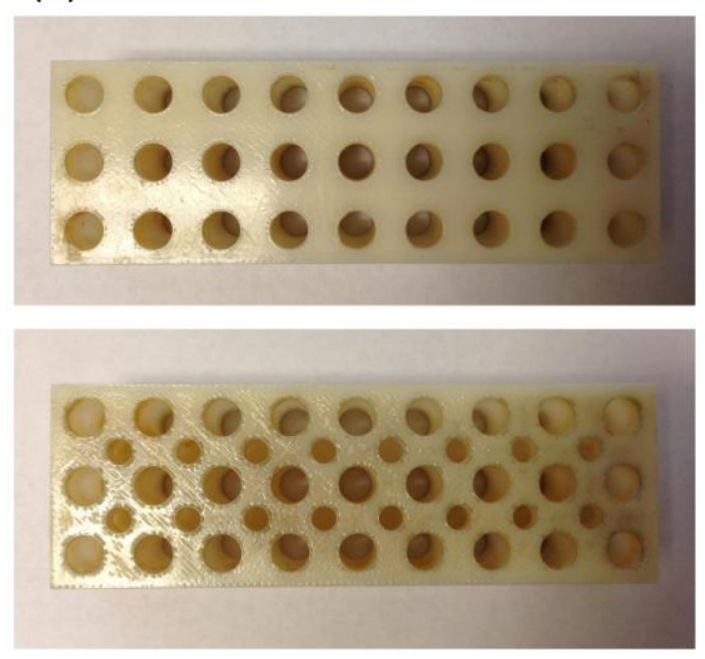

(b)

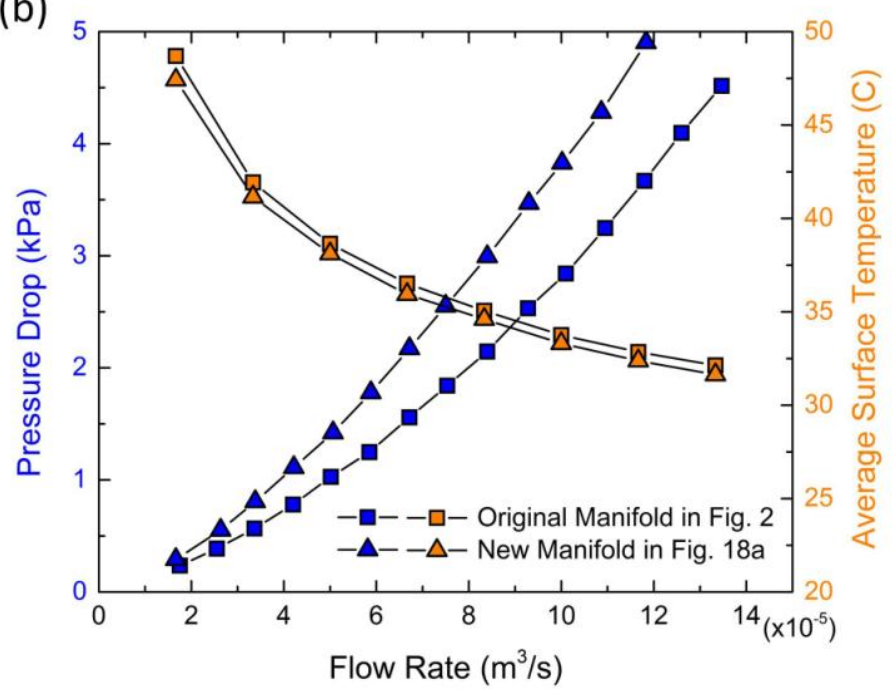

Fig. 18. An alternative design of the manifold in which the outlet hole diameter is only $62.5 \%$ of the inlet hole diameter. The top and bottom surfaces of the manifold are shown in (a) and a comparison of pressure drop and average surface temperature for a $12.7 \mathrm{~mm}$ thick optimized woven block plus a $12.7 \mathrm{~mm}$ thick manifold is shown in (b) using either the original or the new manifold.

\section{Summary and Conclusions}

In an attempt to augment the fluidic and thermal properties inherent in cooling with a 3D weave [1], we designed a new cooling system by combining a 3D printed flow manifold with thinner weaves. The manifold not only creates a novel 3D flow pattern within the weave, it is also capable of dividing a large volume into many small flow unit cells for which properties can be optimized and then extended over very large lateral dimensions. This enhances our ability to tune and enhance cooling systems.

Compared to the case of axial and bifurcated flow through weaves only [1], the new distributed array flow pattern obtained with the weave/manifold system shows very low pressure drops (slightly higher than bifurcated), very low average surface temperatures (slightly higher 
than axial) and by far the smallest temperature variations, given the same overall system thickness. In addition, the volume of the 3D Cu weave is reduced significantly (more than 50\%) with the freed space being taken up by the polymeric flow manifold, which can lower system weight and cost considerably.

To examine the effect of weave thickness given a constant total weave/manifold thickness on system performance, we studied three weave/manifold thickness ratios. The intermediate ratio $(6.4 \mathrm{~mm}$ weave $+19.0 \mathrm{~mm}$ manifold $)$ shows the lowest overall pressure drop, the lowest ratio $(3.2 \mathrm{~mm}$ weave $+22.2 \mathrm{~mm}$ manifold) shows the lowest average surface temperature and the highest ratio $(12.7 \mathrm{~mm}$ weave $+12.7 \mathrm{~mm}$ manifold $)$ shows the lowest temperature variation across the surface. Thus, each ratio demonstrates some advantage, depending on the property considered.

To investigate the effects of the weaves within the weave/manifold system, we studied the performance of three open space/manifold systems by removing the weaves. While the existence of the weave increases the overall pressure drop as expected, it reduces the average surface temperatures and temperature variations significantly. This suggests that a heat exchanger media (woven lattice in this case) is necessary to distribute the heat most effectively into the cooling media.

To account for the practical concern of pumping power, we compared the thermal performance of the various heat exchanger systems at different pumping powers. The weave/manifold system had up to $3 \mathrm{X}$ lower average surface temperatures $\left(T_{s}\right)$ and up to $15 \mathrm{X}$ lower temperature variations $(\Delta T)$ compared to the open space/manifold systems at the same pumping power. This comparison holds for both the standard and optimized weaves, as both architectures showed similar performances at a given pumping power. The weave/manifold 
distributed array system also showed superior thermal performance compared to the axial and bifurcated flow systems, at similar pumping power. In particular, temperature variations were much lower than for the other two systems at low pumping powers.

Lastly, the design of the flow manifold and the heat exchange media can be optimized further. Recent work in topology optimization for convective heat transfer [12] may offer a path towards optimizing geometric features of the manifold and/or the weaves. In fact, the choice of the heat exchange media is not limited to the $3 \mathrm{D}$ woven metallic lattices, but can be broadened to other periodic or graded architectures and can be manufactured using the newest technologies such as 3D printing. The material of the manifold is also subject to change (e.g. to conductive material), and can affect the optimal weave:manifold height ratio. In general, optimization of the manifold should be undertaken with specific manifold and heat exchange materials in mind.

\section{Acknowledgements}

This research was funded by Defense Advanced Research Projects Agency (DARPA) Materials with Controlled Microstructural Architecture (MCMA), under award number W91CRB1010004 (Dr. Judah Goldwasser, Program Manager), and the writing of this manuscript was supported by NSF Grant CMMI-1538367. The authors would like to thank Mr. Daren Ayres for machining test fixtures.

\section{References}

[1] L. Zhao, S.M. Ryan, J.K. Ortega, S. Ha, K.W. Sharp, J.K. Guest, et al., Experimental investigation of 3D woven $\mathrm{Cu}$ lattices for heat exchanger applications, Int. J. Heat Mass 
Transf. 96 (2016) 296-311.

[2] E. a. Browne, G.J. Michna, M.K. Jensen, Y. Peles, Microjet array single-phase and flow boiling heat transfer with R134a, Int. J. Heat Mass Transf. 53 (2010) 5027-5034. doi:10.1016/j.ijheatmasstransfer.2010.07.062.

[3] S. Motakef, M.R. Overholt, Industrial Applications of Thermal Devices with Meso-Scale Features, (2005) 1-15.

[4] M.R. Overholt, A. Mccandless, K.W. Kelly, C.J. Becnel, S. Motakef, Icmm2005-75250 Micro-Jet Arrays for Cooling of Electronic Equipment, Int. Conf. Microchannels Minichannels. (2005) 3-6.

[5] D. Lytle, B.. Webb, Air jet impingement heat transfer at low nozzle-plate spacings, Int. J. Heat Mass Transf. 37 (1994) 1687-1697. doi:10.1016/0017-9310(94)90059-0.

[6] S. V. Garimella, R. a. Rice, Confined and Submerged Liquid Jet Impingement Heat Transfer, J. Heat Transfer. 117 (1995) 871. doi:10.1115/1.2836304.

[7] L. Zhao, S. Ha, K.W. Sharp, A.B. Geltmacher, R.W. Fonda, A.H. Kinsey, et al., Permeability measurements and modeling of topology-optimized metallic 3-D woven lattices, Acta Mater. 81 (2014) 326-336. doi:10.1016/j.actamat.2014.08.037.

[8] Y. Zhang, S. Ha, K. Sharp, J.K. Guest, T.P. Weihs, K.J. Hemker, Fabrication and mechanical characterization of 3D woven Cu lattice materials, Mater. Des. 85 (2015) 743751. doi:10.1016/j.matdes.2015.06.131.

[9] S.J. Kline, F.A. McClintock, Describing Uncertainties in Single-Sample Experiments, Mech. Eng. (Am. Soc. Mech. Eng.). 75 (1953) 3-8.

[10] M. Osanov, J.K. Guest, Topology Optimization for Architected Materials Design, http://dx.doi.org/10.1146/annurev-Matsci-070115-031826. 46 (2016) 211-233.

[11] J.K. Guest, J.H. Prévost, Optimizing multifunctional materials: Design of microstructures for maximized stiffness and fluid permeability, Int. J. Solids Struct. 43 (2006) 7028-7047. doi:10.1016/j.ijsolstr.2006.03.001.

[12] J. Alexandersen, O. Sigmund, N. Aage, International Journal of Heat and Mass Transfer Large scale three-dimensional topology optimisation of heat sinks cooled by natural 
convection, Int. J. Heat Mass Transf. 100 (2016) 876-891. doi:10.1016/j.ijheatmasstransfer.2016.05.013. 